\title{
Assessment of Quality of Life of Patients with Chronic Hepatitis $B$ and $C$ Treated with Pegylated Interferon-alpha
}

\section{Kronik Hepatit B ve Hepatit C'de Pegile İnterferon-alfa Tedavisi Alan Hastalarda Yașam Kalitesinin Değerlendirilmesi}

\author{
(1) Handan ALAY1, (1) Kemalettin ÖZDEN2, (1) Serpil EROL33, 이 Neslihan ÇELIK4, (1) Emine PARLAK2, \\ (1) Mehmet PARLAK2 \\ ${ }^{1}$ Erzurum Nenehatun Maternity Hospital, Clinic of Infectious Diseases and Clinical Microbiology, Erzurum, Turkey \\ ${ }^{2}$ Atatürk University Faculty of Medicine, Department of Infectious Diseases and Clinical Microbiology, Erzurum, Turkey \\ 3/stanbul Haydarpaşa Numune Training and Research Hospital, Clinic of Infectious Diseases and Clinical Microbiology, Istanbul, Turkey \\ 4 University of Health Sciences, Erzurum Bölge Training and Research Hospital, Clinic of Infectious Diseases and Clinical Microbiology, Erzurum, Turkey
}

ABSTRACT

Objectives: It was aimed to evaluate health-related quality of life of patients with non-cirrhotic chronic hepatitis $\mathrm{B}(\mathrm{CHB})$ and chronic hepatitis $\mathrm{C}(\mathrm{CHC})$ during interferon therapy with the standard short form-36 (SF-36).

Materials and Methods: This study included all patients who attended the Atatürk University Faculty of Medicine, Department of Infectious Diseases and Clinical Microbiology and outpatient clinics for treatment between June 2008 and June 2010 and met the inclusion criteria. A socio-demographic data questionnaire and SF36 were administered in all subjects before the interferon therapy and in the third and sixth months of the treatment.

Results: Before the treatment, vitality/energy $(p=0.01)$ and general health $(p=0.01)$ scores in patients with CHB were lower than in controls. In the sixth month of the therapy, physical function $(p=0.03)$, role physical $(p=0.011)$, role emotional $(p=0.003)$ and vitality/energy $(p=0.005)$ scores were significantly lower than in controls. There was a significant difference in physical function $(p=0.006)$, role physical $(p=0.006)$, role emotional $(p=0.001)$ and vitality/energy $(p=0.000005)$ scores before the treatment and physical function $(p=0.006)$, role physical $(p=0.013)$, role emotional $(p=0.001)$, vitality/energy $(p=0.000005)$ and mental health $(p=0.041)$ scores in the third month of the treatment and physical function $(p=0.000008)$, social function $(p=0.005)$, role physical $(p=0.0000008)$, role emotional $(p=0.000007)$, mental health $(p=0.001)$ and vitality/energy $(p=0.000005)$ scores in the sixth month of the treatment between patients with $\mathrm{CHC}$ and controls. Conclusion: Providing guidance and counseling to patients with $\mathrm{CHB}$ and $\mathrm{CHC}$ about their illness and side effects of the drugs will increase health-related quality of life of patients and will adapt them to their treatment.

Keywords: Chronic hepatitis B, chronic hepatitis C, health-related quality of life, interferon therapy
ÖZ

Amaç: Non-sirotik kronik hepatit B (KHB) ve kronik hepatit C'li (KHC) hastalarda interferon tedavisi süresince kısa form 36 (SF-36) standart formunu kullanarak sağlıkla ilgili yaşam kalitesini değerlendirmek amaçlanmıştır.

Gereç ve Yöntemler: Çalıșmaya Haziran 2008 - Haziran 2010 tarihleri tarihleri arasındaki 2 yıllık süre boyunca Atatürk Üniversitesi Tıp Fakültesi, Enfeksiyon Hastlıkları ve Klinik Mikrobiyoloji Anabilim Dalı'na ve polikliniğine başvuran ve çalışmaya dahil edilme kriterlerini karşılayan olgular alındı. Çalışmaya dahil edilen tüm olgulara, interferon tedavisi öncesinde, tedavinin üçüncü ayında ve tedavinin altıncı ayında, sosyo-demografik veri formu ve SF-36 ölçeği uygulandı.

Bulgular: KHB'li hastaların tedavi öncesi enerji $(p=0,01)$ ve genel sağlık $(p=0,01)$ skorlarını; tedavinin altıncı ayında fiziksel fonksiyon $(p=0,03)$, fiziksel rol $(p=0,011)$, emosyonel rol $(p=0,003)$ ve enerji $(p=0,005)$ skorlarını kontrol grubuna göre anlamlı düzeyde daha düşüktü. KHC'li hastaların tedavi öncesi fiziksel fonksiyon $(p=0,006)$, fiziksel rol $(p=0,006)$, emosyonel rol $(p=0,001)$ ve enerji $(p=0,000005)$ skorlarında; tedavinin üçüncü ayında fiziksel fonksiyon $(p=0,006)$, fiziksel rol $(p=0,013)$, emosyonel rol $(p=0,001)$, mental sağlık $(p=0,041)$ ve enerji $(p=0,000005)$ skorlarında; tedavinin altıncı ayında ise fiziksel fonksiyon ( $p=0,000008)$, sosyal fonksiyon $(p=0,005)$, fiziksel rol $(p=0,0000008)$, emosyonel rol $(p=0,000007)$, mental sağlık $(p=0,001)$ ve enerji $(p=0,000005)$ skorlarında kontrol grubuna göre anlamlı düzeyde farklı olduğu saptanmıștır.

Sonuç: Hastalara hastalıkları ve ilaçların yan etkileriyle ilgili rehberlik ve danışmanlık hizmetlerinin verilmesi hastaların yaşam kalitelerini artırıp, tedavi uyumunu sağlayacaktır.

Anahtar Kelimeler: Kronik hepatit B, kronik hepatit C, sağlıkla ilgili yaşam kalitesi, interferon tedavisi

Alay H, Özden K, Erol S, Çelik N, Parlak E, Parlak M. Assessment of Quality of Life of Patients with Chronic Hepatitis B and C Treated with Pegylated Interferonalpha. Viral Hepat J. 2018;24:25-42. 


\section{Introduction}

The two most common viruses capable of causing chronic infection in the liver and associated complications are hepatitis $B$ virus (HBV) and hepatitis C virus (HCV) (1). These are also the most important causes of chronic hepatitis in Turkey and other regions of the world. According to the World Health Organization, approximately 350-400 million people worldwide carry the virus and 1-2 million people a year die of HBV infection or complications (2). $\mathrm{HCV}$ infection is a widespread and severe health problem worldwide. The global prevalence of HCV infection is 3\%, and 210 million people are infected (3). Quality of life is a subjective concept, and difficult to define and measure. Chronic liver disease is generally asymptomatic, but may exhibit systemic symptoms such as fatigue, nausea, pruritus, lack of appetite and psychological disorders. A significant impairment in health-related quality of life ( $\mathrm{HRQoL}$ ) may occur in this patient group (4). The majority of studies of HRQoL in chronic viral hepatitis have been concerned with HCV infection, while the number of studies concerning HBV infection is limited. Several studies of patients infected with HCV have determined a significant decrease in HRQoL compared to controls $(5,6)$. Interferon alpha (IFN- $\alpha$ ) is the first cytokine produced by recombinant DNA technology and is used in the treatment of numerous malignant and non-malignant diseases. Diseases treated using IFN- $\alpha$ include hepatitis B and C. Quality of life of patients with chronic hepatitis $\mathrm{B}(\mathrm{CHB})$ and chronic hepatitis $\mathrm{C}$ $(\mathrm{CHC})$ under IFN therapy is known to be adversely affected (4). The purpose of this study was to evaluate HRQoL scores of naive $\mathrm{CHB}$ and $\mathrm{CHC}$ patients in the infectious diseases and clinical microbiology clinic before pegylated IFN (PEG-IFN) therapy and at the $3^{\text {rd }}$ and $6^{\text {th }}$ months of treatment and to investigate the effect of IFN therapy on quality of life by comparing these with the scores of healthy controls.

\section{Materials and Methods}

\section{Research Type and Sample}

Twenty-eight treatment-naive patients with HBV infection with alanine aminotransferase (ALT) levels twice as high as normal for 6 months, hepatitis B surface antigen $(\mathrm{HBs} A g)+$, hepatitis $B$ e antigen $(\mathrm{HBeAg})+/-$, with $\mathrm{HBV}$-DNA $\geq 10^{4}$ copies/mL and no clinical findings of cirrhosis, and 23 non-cirrhotic $\mathrm{CHC}$ patients, anti-HCV+, with determinable HCV-RNA levels, presenting to and treated in the infectious diseases and clinical microbiology clinic between June 2008 and June 2010 were included in this prospective clinical study. Fifty-one subjects with no underlying chronic disease were enrolled as the control group. All cases were selected from among individuals aged $17-69$ years.

The participants were informed about the study at interviews before commencement, and informed consent was received from all. The study was approved by Atatürk University Faculty of Medicine Ethics Committee (approval number: 65/2008). A sociodemographic data questionnaire was used in order to determine subjects' socio-demographic and disease characteristics, and the 36-Item Short Form-36 (SF-36) Health Survey was administered in order to measure quality of life. The face-to-face interview technique was used for data collection. The forms were administered verbally by a researcher, and the subjects were asked to indicate the option best matching their own circumstances.

\section{Definitions Used in the Research}

Patient group: Treatment-naive non-cirrhotic patients with $\mathrm{HBV}$ infection, with an at least 2-fold increase in ALT levels in the previous 6 months, HBsAg+, HBeAg+/-, HBV-DNA $\geq 10^{4}$ copies/mL with polymerase chain reaction (PCR) and with necroinflammatory activity $\geq 4$ and/or fibrosis $\geq 2$ in liver biopsy, and naive patients diagnosed with chronic non-cirrhotic HCV infection, anti-HCV+ and with HCV-RNA capable of determination with PCR were included in the study. Patients with chronic HBV and HCV infection were started on PEG-IFN $\alpha$-2a therapy.

Control group: Subjects with no underlying chronic disease.

\section{Socio-demographic Data Form}

A socio-demographic form consisting of nine questions was employed to determine subjects' sex, marital status, number of children, place of residence, education level, and occupation.

\section{The 36-Item Short Form Health Survey}

The SF-36 was developed by the Rand Corporation for assessing HRQoL (7). The form has been translated into Turkish and its validity and reliability have been confirmed $(8,9)$. The scale is a generic, self-report outcome measure. It consists of 36 items measuring eight domains-physical functioning, social functioning, physical role limitations, emotional role limitations, mental health, energy/vitality, bodily pain and general health perception. It can evaluate the positive aspects of health status, as well as negative aspects (10). The SF-36 scoring requires a separate guideline. Subdomain score calculation can be performed with a series of procedures (10). Scores range from 0 (worst possible health) to 100 (best possible health), with higher scores indicating a better quality of life. All sections are scored independently (7).

\section{Application Procedure}

Patients presenting to the Atatürk University Faculty of Medicine Infectious Diseases and Clinical Microbiology Clinic between June, 2008 and June, 2010 and meeting the inclusion criteria were enrolled. All subjects enrolled were administered a socio-demographic data questionnaire and the SF-36 before IFN therapy and on the $3^{\text {rd }}$ and $6^{\text {th }}$ months of treatment.

\section{Statistical Analysis}

All the study data were coded numerically and subjected to the One-Way Analysis of Variance and the Mann-Whitney $U$ test in a computer environment using the Statistical Package for Social Sciences (SPSS) v.18.0. A p value of less than 0.05 was considered statistically significant.

\section{Results}

\section{Socio-demographic Characteristics}

Socio-demographic characteristics of the patients are presented in Table 1.

\section{Quality of Life Scores}

Physical functioning, social functioning, physical role limitations, emotional role limitations, mental health, energy/vitality, bodily pain and general health perception scores based on responses to the questions in the SF-36 were obtained for the patient group consisting of patients with $\mathrm{HBV}$ and $\mathrm{HCV}$ and receiving PEG-IFN 
therapy and for the control group consisting of healthy adults. Physical functioning ( $p=0.007)$, physical role limitations $(p=0.008)$, emotional role limitations $(p=0.007)$, vitality/energy $(p=0.001)$, and general health $(p=0.001)$ scores before treatment in the patient group were statistically significantly lower than in the control group. On the $3^{\text {rd }}$ month of treatment, a statistically significant decrease was observed in the physical role limitations $(p=0.03)$, emotional role limitations $(p=0.01)$ and vitality/energy $(p=0.002)$ scores in the patient group. On the $6^{\text {th }}$ month of treatment, a statistically significant decrease was determined in the physical functioning $(p=0.001)$, physical role limitations $(p=0.004)$, emotional role limitations $(p=0.004)$, mental health $(p=0.004)$ and vitality/ energy $(p=0.0003)$ scores. When quality of life scores of patients with $\mathrm{CHB}$ and $\mathrm{CHC}$ were compared with pre-treatment values, a statistically significant decrease was determined in the emotional role limitations scores $(p=0.027)$ in patients with $\mathrm{CHC}$. A statistically significant decrease was also observed in the physical functioning $(p=0.042)$ and social functioning $(p=0.042)$ scores in patients with $\mathrm{CHC}$ on the $6^{\text {th }}$ month of treatment. When we compared the pre- treatment HRQoL scores of patients with $\mathrm{CHB}$ and controls, we determined statistically significantly lower vitality/energy $(p=0.01)$ and general health $(p=0.01)$ scores in the patient group than in controls. On the $6^{\text {th }}$ month of treatment, statistically significant differences were determined in the physical functioning $(p=0.03)$, physical role limitations $(p=0.011)$, emotional role limitations $(p=0.003)$ and vitality/energy $(p=0.006)$ scores in patients with $\mathrm{CHB}$. When we compared the pre-treatment HROoL scores of patients with $\mathrm{CHC}$ and controls, we determined statistically significantly lower physical functioning $(p=0.006)$, physical role limitations $(p=0.006)$, emotional role limitations $(p=0.001)$, vitality/energy $(p=0.000005)$, and general health $(p=0.003)$ scores in patients with chronic hepatitis. A statistically significant decrease was determined in physical functioning $(p=0.006)$, physical role limitations $(p=0.013)$, emotional role limitations $(p=0.001)$, mental health $(p=0.041)$ and vitality/energy $(p=0.000005)$ scores in patients with hepatitis $C$ on the $3^{\text {rd }}$ month of treatment. On the $6^{\text {th }}$ month of treatment, statistically significant decreases were observed in physical functioning ( $p=0.000008)$, social functioning $(p=0.005)$, physical role

\begin{tabular}{|c|c|c|c|c|c|c|c|c|c|c|c|c|c|}
\hline & & \multicolumn{8}{|c|}{ Groups } & \multirow{2}{*}{\multicolumn{4}{|c|}{$\mathbf{p}$}} \\
\hline & & \multicolumn{2}{|c|}{ Patient (all) } & \multicolumn{2}{|l|}{ CHB } & \multicolumn{2}{|l|}{$\mathrm{CHC}$} & \multicolumn{2}{|l|}{ Control } & & & & \\
\hline & & Number & $\%$ & Number & $\%$ & Number & $\%$ & Number & $\%$ & $\begin{array}{l}\text { Patient- } \\
\text { control }\end{array}$ & $\begin{array}{l}\text { CHB- } \\
\text { CHC }\end{array}$ & \begin{tabular}{|l|} 
CHB- \\
control
\end{tabular} & $\begin{array}{l}\text { CHC- } \\
\text { control }\end{array}$ \\
\hline \multirow{2}{*}{ Gender } & Female & 24 & 47.1 & 8 & 28.6 & 16 & 69.6 & 24 & 47.1 & \multirow{2}{*}{1} & \multirow{2}{*}{0.778} & \multirow{2}{*}{0.108} & \multirow{2}{*}{0.068} \\
\hline & Male & 27 & 52.9 & 20 & 71.4 & 7 & 30.4 & 27 & 52.9 & & & & \\
\hline \multirow{3}{*}{$\begin{array}{l}\text { Marriage } \\
\text { status }\end{array}$} & Married & 37 & 72.5 & 20 & 71.4 & 17 & 73.9 & 42 & 82.4 & \multirow{3}{*}{0.348} & \multirow{3}{*}{0.691} & \multirow{3}{*}{0.574} & \multirow{3}{*}{0.321} \\
\hline & Single & 12 & 23.5 & 8 & 28.6 & 4 & 17.4 & 7 & 13.7 & & & & \\
\hline & Widowed & 2 & 3.9 & 0 & 0 & 2 & 8.7 & 2 & 3.9 & & & & \\
\hline \multirow{3}{*}{$\begin{array}{l}\text { Place of } \\
\text { residence }\end{array}$} & Province/city & 26 & 51 & 13 & 46.4 & 13 & 56.5 & 17 & 33.3 & \multirow{3}{*}{0.901} & \multirow{3}{*}{0.644} & \multirow{3}{*}{0.858} & \multirow{3}{*}{0.675} \\
\hline & District & 9 & 17.6 & 6 & 21.4 & 3 & 13 & 26 & 51 & & & & \\
\hline & Town/village & 16 & 31.4 & 9 & 32.2 & 7 & 30.5 & 8 & 15.7 & & & & \\
\hline \multirow{5}{*}{$\begin{array}{l}\text { Educational } \\
\text { status }\end{array}$} & Illiterate & 9 & 17.6 & 2 & 7.1 & 7 & 30.4 & 6 & 11.8 & \multirow{5}{*}{0.862} & \multirow{5}{*}{0.082} & \multirow{5}{*}{0.413} & \multirow{5}{*}{0.216} \\
\hline & Literate & 5 & 9.8 & 2 & 7.1 & 3 & 13 & 10 & 19.6 & & & & \\
\hline & $\begin{array}{l}\text { Elementary } \\
\text { school }\end{array}$ & 22 & 43.2 & 15 & 53.7 & 7 & 30.4 & 19 & 37.3 & & & & \\
\hline & High school & 11 & 21.6 & 7 & 25 & 4 & 17.5 & 12 & 23.5 & & & & \\
\hline & University & 4 & 7.8 & 2 & 7.1 & 2 & 8.7 & 4 & 7.8 & & & & \\
\hline \multirow{8}{*}{ Occupations } & Unemployed & 2 & 3.9 & 2 & 7.1 & 0 & 0 & 2 & 3.9 & \multirow{8}{*}{0.507} & \multirow{8}{*}{0.247} & \multirow{8}{*}{0.237} & \\
\hline & $\begin{array}{l}\text { Seasonal } \\
\text { agricultural } \\
\text { worker }\end{array}$ & 1 & 2 & 0 & 0 & 1 & 4.3 & 2 & 3.9 & & & & \\
\hline & Employee & 6 & 11.8 & 3 & 10.7 & 3 & 13 & 8 & 15.7 & & & & \\
\hline & Housewife & 20 & 39.2 & 7 & 25 & 13 & 56.5 & 18 & 35.3 & & & & 0.848 \\
\hline & Worker & 2 & 3.9 & 2 & 7.1 & 0 & 0 & 7 & 13.7 & & & & \\
\hline & $\begin{array}{l}\text { Self- } \\
\text { employment }\end{array}$ & 10 & 19.6 & 8 & 28.6 & 2 & 8.7 & 6 & 11.8 & & & & \\
\hline & Student & 5 & 9.8 & 2 & 7.1 & 3 & 13 & 2 & 3.9 & & & & \\
\hline & Other & 5 & 9.8 & 4 & 14.3 & 1 & 4.3 & 6 & 11.8 & & & & \\
\hline
\end{tabular}


limitations $(p=0.0000008)$, emotional role limitations $(p=0.000007)$, mental health $(p=0.001)$ and vitality/energy $(p=0.000005)$ scores. Mean values, standard deviation and $p$ values obtained for all groups in the study before and after 3 and 6 months of treatment are shown in Table 2. Distribution of SF-36 scores by socio-demographic properties of patients is summarized in Table 3. Distribution of SF-36 scores by socio-demographic properties of $\mathrm{CHB}$ patients in treatment periods is summarized in Table 4. Distribution of SF-36 scores by socio-demographic properties of $\mathrm{CHC}$ patients in treatment periods is summarized in Table 5 .

\section{Discussion}

Patients with chronic hepatitis are generally asymptomatic, but may also exhibit systemic symptoms, such as fatigue, nausea, pruritus, lack of appetite and psychological disorders. A significant impairment in HRQoL may occur in this patient group. The majority of studies of HRQoL in chronic viral hepatitis have been concerned with HCV infection, while the number of studies concerning HBV infection is limited (4). HRQoL of patients with chronic hepatitis may vary depending on their socio-demographic characteristics. Numerous studies have shown that sex, marital status, education level, occupation and place of residence affect HRQoL of HBV- and HCV-infected patients. In agreement with the previous literature, we determined a significant decrease in female patients with chronic hepatitis $(11,12,13,14,15,16,17,18$, 19,20). Women with chronic disease are known to receive less social support than men in many parts of the world. In addition, they generally receive medical care later than males; they either have to work, or else have to resume their responsibilities without being fully recovered (21). These may all account for the decrease in HRQoL of female patients with chronic hepatitis. In agreement with previous studies, we determined lower HRQoL scores in individuals infected with $\mathrm{HCV}(22,23)$. Being married and having social and individual responsibilities may affect HRQoL. No significant change in $\mathrm{HRQOL}$ and only a weak correlation between marital status and HRQoL was observed in married patients

\begin{tabular}{|c|c|c|c|c|c|c|c|c|}
\hline \multicolumn{9}{|c|}{ Before treatment } \\
\hline & \multicolumn{2}{|c|}{ Physical functioning } & \multicolumn{2}{|c|}{ Social functioning } & \multicolumn{2}{|c|}{ Physical role } & \multicolumn{2}{|c|}{ Emotional role } \\
\hline & Avg. \pm SD & Median; IQR & Avg. \pm SD & Median; IQR & Avg. \pm SD & Median; IQR & Avg. \pm SD & Median; IQR \\
\hline Patient & $63.1 \pm 34$ & 0.007 & $81.3 \pm 25.5$ & 0.472 & $43.6 \pm 46.1$ & 0.008 & $36.8 \pm 34.8$ & 0.007 \\
\hline $\mathrm{CHC}$ & $56.3 \pm 33.5$ & $90 ; 30$ & $80.2 \pm 24.8$ & $88.9 ; 22.2$ & $32.6 \pm 43.6$ & $100 ; 75$ & $25 \pm 32.9$ & $75 ; 25$ \\
\hline $\mathrm{CHB}$ & $68.8 \pm 34$ & 0.05 & $82.1 \pm 26.4$ & 0.613 & $52.7 \pm 46.8$ & 0.168 & $46.4 \pm 33.8$ & 0.28 \\
\hline Control & $81.8 \pm 23.3$ & $82.5 ; 48.75$ & $85 \pm 17.8$ & $100 ; 30.56$ & $67.2 \pm 42$ & $62.5 ; 100$ & $55 \pm 29.2$ & $25 ; 25$ \\
\hline $\mathrm{CHC}$ & $56.3 \pm 33.5$ & 0.006 & $80.2 \pm 24.8$ & 0.451 & $32.6 \pm 43.6$ & 0.006 & $25 \pm 32.9$ & 0.001 \\
\hline Control & $81.8 \pm 23.3$ & $80 ; 55$ & $85 \pm 17.8$ & $100 ; 44.44$ & $67.2 \pm 42$ & $25 ; 100$ & $55 \pm 29.2$ & $25 ; 75$ \\
\hline $\mathrm{CHB}$ & $78 \pm 30$ & 0.122 & $80.2 \pm 29.5$ & 0.575 & $52.7 \pm 46.3$ & 0.444 & $44.6 \pm 36.2$ & 0.173 \\
\hline $\mathrm{CHC}$ & $58.3 \pm 57.8$ & $90 ; 30$ & $75.4 \pm 31.1$ & $88.9 ; 22.22$ & $42.4 \pm 48.5$ & $100 ; 75$ & $30.4 \pm 36.9$ & $75 ; 25$ \\
\hline $\mathrm{CHB}$ & $78 \pm 30$ & 0.573 & $80.2 \pm 29.5$ & 0.389 & $52.7 \pm 46.3$ & 0.168 & $44.6 \pm 36.2$ & 0.192 \\
\hline Control & $81.8 \pm 23.3$ & $95 ; 41.25$ & $85 \pm 17.8$ & $100 ; 41.67$ & $67.2 \pm 42$ & $62.5 ; 100$ & $55 \pm 29.2$ & $75 ; 75$ \\
\hline $\mathrm{CHC}$ & $58.3 \pm 57.8$ & 0.006 & $75.4 \pm 31.1$ & 0.097 & $42.4 \pm 48.5$ & 0.013 & $30.4 \pm 36.9$ & 0.001 \\
\hline Control & $81.8 \pm 23.3$ & $65 ; 90$ & $85 \pm 17.8$ & $100 ; 44.44$ & $67.2 \pm 42$ & $0 ; 100$ & $55 \pm 29.2$ & $0 ; 75$ \\
\hline \multicolumn{9}{|c|}{$6^{\text {th }}$ months of treatment } \\
\hline Patient & $59.2 \pm 31.6$ & 0.001 & $76.7 \pm 28.8$ & 0.109 & $31.9 \pm 42.4$ & 0.004 & $23.5 \pm 32.9$ & 0.004 \\
\hline Control & $81.8 \pm 23.3$ & $55 ; 55$ & $85 \pm 17.8$ & $77.77 ; 55.56$ & $67.2 \pm 42$ & $0 ; 25$ & $55 \pm 29.2$ & $0 ; 25$ \\
\hline
\end{tabular}


with chronic viral hepatitis B in previous studies. In the present study also, no significant changes in HRQoL were determined in this patient group. A low education level is another demographic characteristic that affects HRQoL. As also shown in several studies, HROoL was statistically significantly affected before and during treatment in our chronic hepatitis patients with a low level of education $(13,16)$. Housewives have been determined to have the lowest scores and clerical workers the highest in all areas of HRQoL (17). Low physical functioning, physical role limitations, general health and emotional role limitations scores have been determined among unemployed patients with $\mathrm{CHB}$ and $\mathrm{CHC}$ (13). In our study, pre-treatment physical functioning and $3^{\text {rd }}$ month physical role limitations scores in patients with $\mathrm{CHB}$ were lower among housewives. Among the patients with $\mathrm{CHC}$, a significant decrease was observed only in the mental health scores in agricultural workers at the $6^{\text {th }}$ month of treatment. The effect of IFN therapy in terms of occupations of patients with chronic viral hepatitis is unclear, and no benchmark has been determined.
However, the numerous side-effects of IFN and ribavirin may be described as an adverse physical impact. Lam et al. (11) showed a significant level of variation in HRQol scores in patients with $\mathrm{CHB}$ in the categories of physical role limitations, bodily pain, energy/vitality, social functioning and emotional role limitations. In a similar study of patients with hepatitis B and C and healthy controls, Ozkan et al. (13) observed a particularly significant decrease in the physical functioning and mental health domains in patients with HBV infection compared to controls. Several studies have reported lower HRQoL scores in patients with HBV and HCV infection compared to healthy controls $(11,12,13,24,25)$. In the present study, we determined lower HRQoL scores in patients with HBV infection compared to healthy controls. Patients with $\mathrm{CHC}$ have more severe and more frequent symptoms of musculoskeletal pain, malaise and fatigue compared with other forms of chronic liver disease $(14,26)$. Several studies have shown a decrease in HRQoL scores in all categories in patients with hepatitis C compared to controls $(12,13,22,24)$. In this

\begin{tabular}{|c|c|c|c|c|c|c|c|c|}
\hline \multicolumn{9}{|c|}{ Before treatment } \\
\hline & \multicolumn{2}{|c|}{ Mental health } & \multicolumn{2}{|c|}{ Energy } & \multicolumn{2}{|c|}{ Bodily pain } & \multicolumn{2}{|c|}{ General health } \\
\hline & & $\mathbf{p}$ & & $\mathrm{p}$ & & $\mathbf{p}$ & & $\mathbf{p}$ \\
\hline & Avg . \pm SD & Median; IQR & Avg. \pm SD & Median; IQR & Avg. \pm SD & Median; IQR & Avg. \pm SD & Median; IQR \\
\hline Patient & $59.8 \pm 23.3$ & 0.37 & $39.1 \pm 29.8$ & 0.001 & $59.5 \pm 31.8$ & 0.446 & $45.5 \pm 21$ & 0.001 \\
\hline Control & $63.8 \pm 21.5$ & $60 ; 40$ & $57.9 \pm 22.4$ & $30 ; 60$ & $63.8 \pm 20.5$ & $55.55 ; 55.55$ & $59.9 \pm 18.8$ & $46 ; 27.5$ \\
\hline $\mathrm{CHB}$ & $62.9 \pm 24.2$ & 0.3 & $41.6 \pm 33.9$ & 0.516 & $66.3 \pm 33.5$ & 0.093 & $47.1 \pm 22.3$ & 0.562 \\
\hline $\mathrm{CHC}$ & $56 \pm 21.9$ & $64 ; 28$ & $36.1 \pm 24.2$ & $60 ; 30$ & $54.2 \pm 28.2$ & $66.7 ; 33.4$ & $43.6 \pm 19.6$ & $57.5 ; 20$ \\
\hline $\mathrm{CHB}$ & $62.9 \pm 24.2$ & 0.865 & $41.6 \pm 33.9$ & 0.01 & $66.3 \pm 33.5$ & 0.712 & $47.1 \pm 22.3$ & 0.01 \\
\hline Control & $63.8 \pm 21.5$ & $68 ; 36$ & $57.9 \pm 22.4$ & $30 ; 63.75$ & $63.8 \pm 20.5$ & $77.77 ; 66.67$ & $59.9 \pm 18.8$ & $47.25 ; 19.75$ \\
\hline $\mathrm{CHC}$ & $56 \pm 21.9$ & 0.284 & $36.1 \pm 24.2$ & 0.000005 & $54.2 \pm 28.2$ & 0.132 & $43.6 \pm 19.6$ & 0.003 \\
\hline Control & $63.8 \pm 21.5$ & $60 ; 44$ & $57.9 \pm 22.4$ & $30 ; 40$ & $63.8 \pm 20.5$ & $44.44 ; 44.44$ & $59.9 \pm 18.8$ & $45 ; 32.5$ \\
\hline \multicolumn{9}{|c|}{$3^{\text {rd }}$ months of treatment } \\
\hline Patient & $56.9 \pm 24.4$ & 0.127 & $41 \pm 30$ & 0.002 & $61 \pm 29$ & 0.621 & $54.8 \pm 24$ & 0.242 \\
\hline Control & $63.8 \pm 21.5$ & $64 ; 28$ & $57.9 \pm 22.4$ & $45 ; 50$ & $63.8 \pm 20.5$ & $66.66 ; 44.44$ & $59.9 \pm 18.8$ & $53.5 ; 35$ \\
\hline $\mathrm{CHB}$ & $60.7 \pm 25$ & 0.227 & $45.9 \pm 29.9$ & 0.2 & $65.9 \pm 29.8$ & 0.189 & $56.2 \pm 23.8$ & 0.642 \\
\hline $\mathrm{CHC}$ & $52.3 \pm 23.4$ & $64 ; 28$ & $35 \pm 29.7$ & $60 ; 30$ & $55.1 \pm 27.5$ & $66.7 ; 33.4$ & $53 \pm 24.5$ & $57.5 ; 20$ \\
\hline $\mathrm{CHB}$ & $60.7 \pm 25$ & 0.569 & $45.9 \pm 29.9$ & 0.06 & $65.9 \pm 29.8$ & 0.758 & $56.2 \pm 23.8$ & 0.476 \\
\hline Control & $63.8 \pm 21.5$ & $64 ; 24$ & $57.9 \pm 22.4$ & $50 ; 50$ & $63.8 \pm 20.5$ & $72.22 ; 55.56$ & $59.9 \pm 18.8$ & $54.75 ; 35$ \\
\hline $\mathrm{CHC}$ & $52.3 \pm 23.4$ & 0.041 & $35 \pm 29.7$ & 0.000005 & $55.1 \pm 27.5$ & 0.229 & $53 \pm 24.5$ & 0.149 \\
\hline Control & $63.8 \pm 21.5$ & $52 ; 40$ & $57.9 \pm 22.4$ & $40 ; 60$ & $63.8 \pm 20.5$ & $44.44 ; 55.56$ & $59.9 \pm 18.8$ & $53.5 ; 35$ \\
\hline \multicolumn{9}{|c|}{$6^{\text {th }}$ months of treatment } \\
\hline Patient & $50.9 \pm 20.6$ & 0.004 & $35.6 \pm 28$ & 0.0003 & $52.7 \pm 32.5$ & 0.05 & $56.5 \pm 24.8$ & 0.439 \\
\hline Control & $63.8 \pm 21.5$ & $52 ; 32$ & $57.9 \pm 22.4$ & $40 ; 100$ & $63.8 \pm 20.5$ & $55.55 ; 55.56$ & $59.9 \pm 18.8$ & $62.5 ; 41$ \\
\hline $\mathrm{CHB}$ & $56 \pm 20.9$ & 0.05 & $39.5 \pm 28.4$ & 0.279 & $54.4 \pm 32.1$ & 0.695 & $57.2 \pm 26$ & 0.833 \\
\hline $\mathrm{CHC}$ & $44.7 \pm 18.8$ & $64 ; 28$ & $30.9 \pm 27.3$ & $60 ; 30$ & $50.7 \pm 33.6$ & $66.7 ; 33.4$ & $55.7 \pm 23.9$ & $57.5 ; 20$ \\
\hline $\mathrm{CHB}$ & $56 \pm 20.9$ & 0.149 & $39.5 \pm 28.4$ & 0.006 & $54.4 \pm 32.1$ & 0.153 & $57.2 \pm 26$ & 0.6 \\
\hline Control & $63.8 \pm 21.5$ & $60 ; 23$ & $57.9 \pm 22.4$ & $45 ; 50$ & $63.8 \pm 20.5$ & $50 ; 61.11$ & $59.9 \pm 18.8$ & $66 ; 41.88$ \\
\hline $\mathrm{CHC}$ & $44.7 \pm 18.8$ & 0.001 & $30.9 \pm 27.3$ & 0.000005 & $50.7 \pm 33.6$ & 0.063 & $55.7 \pm 23.9$ & 0.352 \\
\hline Control & $63.8 \pm 21.5$ & $48 ; 32$ & $57.9 \pm 22.4$ & $30 ; 50$ & $63.8 \pm 20.5$ & $55.55 ; 55.56$ & $59.9 \pm 18.8$ & $60 ; 40$ \\
\hline
\end{tabular}




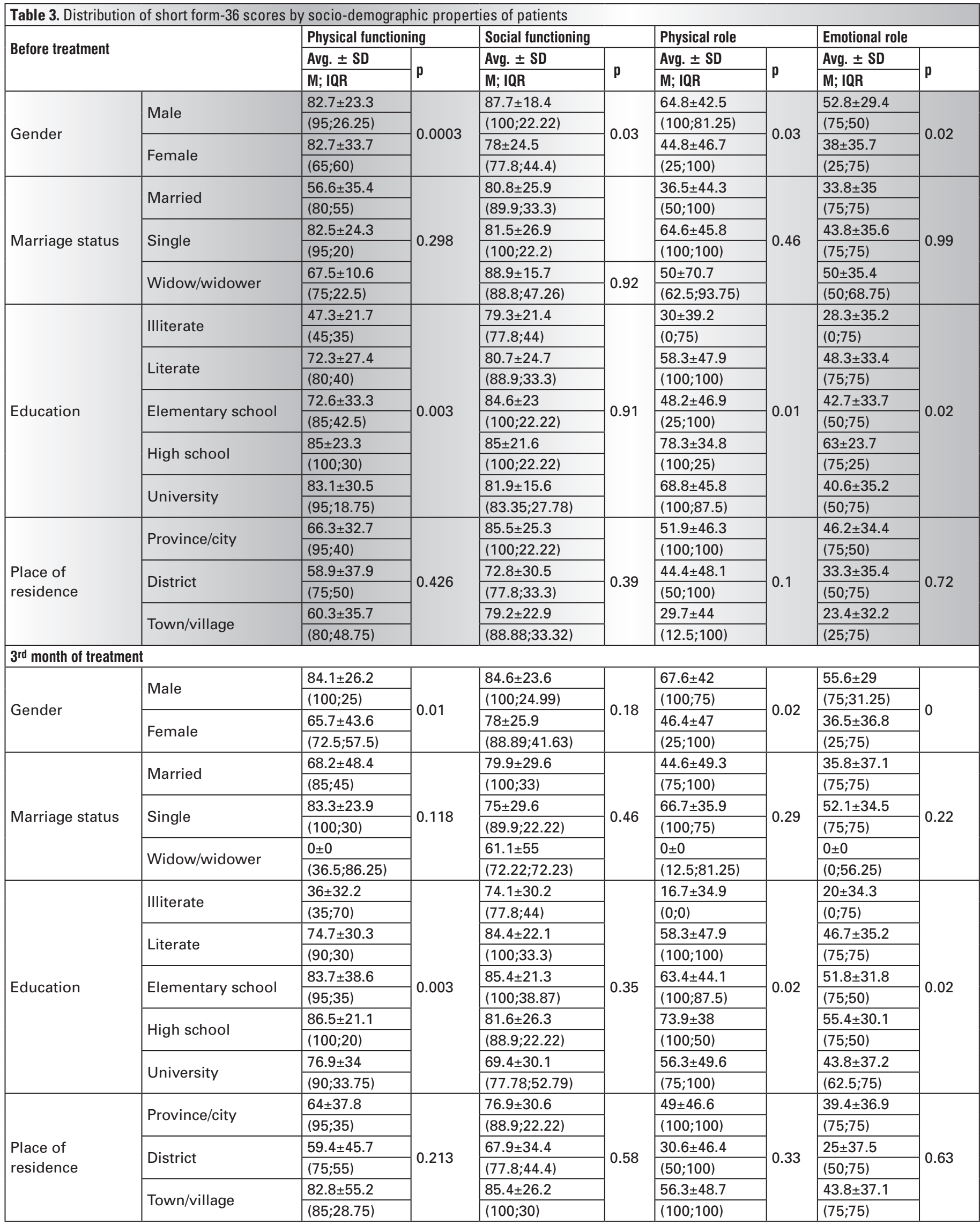




\begin{tabular}{|c|c|c|c|c|c|c|c|c|c|}
\hline \multicolumn{10}{|l|}{ Table 3. Continued } \\
\hline \multirow{3}{*}{\multicolumn{2}{|c|}{ Before treatment }} & \multicolumn{2}{|l|}{ Mental health } & \multicolumn{2}{|l|}{ Energy } & \multicolumn{2}{|l|}{ Bodily pain } & \multicolumn{2}{|l|}{ General health } \\
\hline & & \multirow{2}{*}{\begin{tabular}{|l|} 
Avg. \pm SD \\
M; IQR \\
\end{tabular}} & \multirow{2}{*}{$p$} & Avg. \pm SD & \multirow[b]{2}{*}{$p$} & Avg. \pm SD & \multirow{2}{*}{$p$} & Avg. \pm SD & \multirow{2}{*}{$p$} \\
\hline & & & & M; IQR & & M; IQR & & M; IQR & \\
\hline \multirow{4}{*}{ Gender } & \multirow{2}{*}{ Male } & $65.1 \pm 20.5$ & \multirow{4}{*}{0.11} & $53.2 \pm 28.5$ & \multirow{4}{*}{0.07} & $69.5 \pm 24.1$ & \multirow{4}{*}{0} & $57.5 \pm 20.9$ & \\
\hline & & $(68 ; 28)$ & & $(50 ; 45)$ & & $(77.77 ; 47.22)$ & & $(55.5 ; 24.13)$ & (ח0 \\
\hline & & $58 \pm 24$ & & $43.2 \pm 26.4$ & & $52.8 \pm 27$ & & $47.4 \pm 20.2$ & 0.02 \\
\hline & Female & $(60 ; 40)$ & & $(40 ; 43.75)$ & & $(55.57 ; 44.44)$ & & $(48.5 ; 33)$ & \\
\hline & & $56.1 \pm 22.5$ & & $30.9 \pm 26.7$ & & $55 \pm 31.7$ & & $43.9 \pm 21.1$ & \\
\hline & Married & \begin{tabular}{|l}
$(60 ; 36)$ \\
\end{tabular} & & $\begin{array}{l}(50 ; 50) \\
\end{array}$ & & $(55.6 ; 33.4)$ & & \begin{tabular}{|l}
$(52.5 ; 26)$ \\
\end{tabular} & \\
\hline & & $69.7 \pm 25.3$ & & $59.2 \pm 29.1$ & & $74.1 \pm 30.1$ & & $50 \pm 22.2$ & \\
\hline Marriage status & Single & $(72 ; 44)$ & 0.57 & $(70 ; 50)$ & 0.08 & $(78.8 ; 44.44)$ & 0.03 & $(48.5 ; 22.5)$ & 0.88 \\
\hline & & $68 \pm 0$ & & $70 \pm 14.1$ & & $55.6 \pm 31.4$ & & $48.8 \pm 15.9$ & \\
\hline & Widow/widower & $(68 ; 6)$ & & \begin{tabular}{|l|}
$(67.5 ; 18.75)$ \\
\end{tabular} & & \begin{tabular}{|l}
$(38.86 ; 44.45)$ \\
\end{tabular} & & $(55 ; 22)$ & \\
\hline & & $49.6 \pm 19.2$ & & $38.7 \pm 24.4$ & & $43.7 \pm 23.9$ & & $43.4 \pm 20.2$ & \\
\hline & Illiterate & $(52 ; 28)$ & & $(40 ; 45)$ & & $(44.44 ; 44.5)$ & & $(38.8 ; 45)$ & \\
\hline & & $65.1 \pm 22.1$ & & $53.3 \pm 28.2$ & & $63 \pm 20.4$ & & $54.8 \pm 15.9$ & \\
\hline & Literate & $(68 ; 36)$ & & $(55 ; 55)$ & & $(77.77 ; 33.4)$ & & \begin{tabular}{|l}
$(52.5 ; 17.5)$ \\
\end{tabular} & \\
\hline & & $64.5 \pm 22.3$ & & $46.6 \pm 30.4$ & & $62.9 \pm 27.8$ & & $52.6 \pm 22.1$ & \\
\hline Education & Elementary school & $(68 ; 38)$ & 0.25 & $(40 ; 55)$ & 0.48 & $(55.6 ; 38.92)$ & 0.56 & \begin{tabular}{|l|}
$(53.5 ; 24.75)$ \\
\end{tabular} & 0.38 \\
\hline & & $63 \pm 22.1$ & & $53.3 \pm 27.3$ & & $69.6 \pm 27.1$ & & $55.3 \pm 24.5$ & \\
\hline & High school & \begin{tabular}{|l|}
$(38 ; 28)$ \\
\end{tabular} & & $(50 ; 30)$ & & $(66.7 ; 44.44)$ & & $(56 ; 21)$ & \\
\hline & & $61 \pm 26.8$ & & $54.4 \pm 20.1$ & & $63.9 \pm 25.7$ & & $59.4 \pm 12.3$ & \\
\hline & University & $(72 ; 43)$ & & \begin{tabular}{|l}
$(57.5 ; 36.25)$ \\
\end{tabular} & & $(55.57 ; 50.04)$ & & \begin{tabular}{|l}
$(48.8 ; 17)$ \\
\end{tabular} & \\
\hline & & $60.3 \pm 24.1$ & & $42.7 \pm 29.5$ & & $65.4 \pm 32.3$ & & $49.4 \pm 21.3$ & \\
\hline & Province/city & \begin{tabular}{|l}
$(68 ; 40)$ \\
\end{tabular} & & $\begin{array}{l}(50 ; 40) \\
\end{array}$ & & \begin{tabular}{|l}
$(77.77 ; 44.44)$ \\
\end{tabular} & & $(53.5 ; 28.5)$ & \\
\hline Place of & District & $56.4 \pm 22.8$ & 036 & $39.4 \pm 39.1$ & 0 & $65.4 \pm 32.1$ & 01 & $44.2 \pm 28$ & (חم \\
\hline residence & District & $(60 ; 28)$ & 0.36 & $(50 ; 45)$ & 0.44 & $(55.6 ; 33.4)$ & 0.1 & $(55 ; 26)$ & 0.09 \\
\hline & & $60.8 \pm 23.4$ & & $33.1 \pm 25$ & & $46.5 \pm 28.8$ & & $39.8 \pm 15.2$ & \\
\hline & lown/village & $(66 ; 35)$ & & \begin{tabular}{|l|}
$(47.5 ; 55)$ \\
\end{tabular} & & \begin{tabular}{|l|}
$(50 ; 44.47)$ \\
\end{tabular} & & $(46.75 ; 15.5)$ & \\
\hline $3^{\text {rd }}$ month of treatm & & & & & & & & & \\
\hline & & $60.2 \pm 24.4$ & & $52.5 \pm 27.6$ & & $69.8 \pm 22.3$ & & $60.1 \pm 22.3$ & \\
\hline & Male & $(66 ; 32)$ & & \begin{tabular}{|l}
$(52.5 ; 32.5)$ \\
\end{tabular} & & \begin{tabular}{|l|}
$(77.77 ; 36.11)$ \\
\end{tabular} & 0 & $(60 ; 31)$ & \\
\hline Gender & & $60.5 \pm 21.9$ & 0.95 & $46 \pm 27.7$ & 0.24 & $54.2 \pm 25.6$ & 0 & $54.2 \pm 20.4$ & 0.17 \\
\hline & Female & \begin{tabular}{|l}
$(64 ; 26)$ \\
\end{tabular} & & $(50 ; 38.75)$ & & $(55.6 ; 55.56)$ & & \begin{tabular}{|l}
$(52.25 ; 24.38)$ \\
\end{tabular} & \\
\hline & Married & $54.7 \pm 24.3$ & & $38.6 \pm 29.9$ & & $59.5 \pm 29.4$ & & $55.5 \pm 24.2$ & \\
\hline & Married & $(64 ; 24)$ & & $(50 ; 40)$ & & $(55.6 ; 33.36)$ & & $(57.5 ; 28.5)$ & \\
\hline & & $61.7 \pm 26.6$ & & $52.5 \pm 29$ & & $70.4 \pm 26.9$ & & $55 \pm 24.6$ & \\
\hline Marriage status & Single & $(64 ; 48)$ & 0.75 & $(55 ; 50)$ & 0.7 & \begin{tabular}{|l|}
$(77.77 ; 22.22)$ \\
\end{tabular} & 0.01 & $(51 ; 16)$ & 0.71 \\
\hline & & $70 \pm 2.8$ & & $15 \pm 21.2$ & & $33.3 \pm 15.7$ & & $40.5 \pm 18.4$ & \\
\hline & Widow/widower & $(70 ; 7)$ & & (43;63.75) & & \begin{tabular}{|l|}
$(32.33 ; 22.21)$ \\
\end{tabular} & & \begin{tabular}{|l|}
$(51.75 ; 27.88)$ \\
\end{tabular} & \\
\hline & & $54.1 \pm 21.4$ & & $32 \pm 24.8$ & & $43.7 \pm 24.7$ & & $46.4 \pm 20.2$ & \\
\hline & Illiterate & $(56 ; 24)$ & & $(30 ; 50)$ & & $(44.44 ; 44.48)$ & & $(42.5 ; 27.5)$ & \\
\hline & I iterate & $66.9 \pm 22$ & & \begin{tabular}{|l|}
$55.7 \pm 28.5$ \\
\end{tabular} & & $60.7 \pm 23$ & & $60.7 \pm 15.6$ & \\
\hline & Literate & $(72 ; 28)$ & & $(60 ; 35)$ & & \begin{tabular}{|l|}
$(66.66 ; 33.4)$ \\
\end{tabular} & & $(57.5 ; 22.5)$ & \\
\hline Edusation & & $63 \pm 22.4$ & 036 & $52.9 \pm 27.3$ & 003 & $66.4 \pm 25.3$ & 001 & $58.9 \pm 22.4$ & 032 \\
\hline Education & Elementary school & $(64 ; 30)$ & 0.36 & $(60 ; 45)$ & 0.03 & $(66.66 ; 44.46)$ & 0.01 & $(60 ; 28.5)$ & 0.33 \\
\hline & High ophol & $58.8 \pm 25.1$ & & $55.2 \pm 26.9$ & & $69.6 \pm 21$ & & $59.1 \pm 24$ & \\
\hline & High school & \begin{tabular}{|l|}
$(68 ; 32)$ \\
\end{tabular} & & \begin{tabular}{|l|}
$(60 ; 30)$ \\
\end{tabular} & & \begin{tabular}{|l|}
$(66.7 ; 33.29)$ \\
\end{tabular} & & \begin{tabular}{|l|}
$(56 ; 16)$ \\
\end{tabular} & \\
\hline & & $50.5 \pm 26$ & & $36.3 \pm 23.7$ & & $59.7 \pm 26.5$ & & $58.4 \pm 20.3$ & \\
\hline & University & $(58 ; 49)$ & & $(45 ; 43.75)$ & & $(50 ; 41.71)$ & & \begin{tabular}{|l}
$(49.25 ; 42.63)$ \\
\end{tabular} & \\
\hline & Provino & $58.6 \pm 25.8$ & & $37.9 \pm 31.1$ & & $61.5 \pm 26.5$ & & $54.9 \pm 23.8$ & \\
\hline & Province/city & $(72 ; 32)$ & & \begin{tabular}{|l|}
$(50 ; 45)$ \\
\end{tabular} & & $(66.66 ; 33.36)$ & & \begin{tabular}{|l|}
$(53.5 ; 21)$ \\
\end{tabular} & \\
\hline Place of & Distrist & $47.6 \pm 23.9$ & 026 & $37.8 \pm 29.9$ & 019 & $63 \pm 36.9$ & 051 & $46.4 \pm 25.3$ & 067 \\
\hline residence & District & $(52 ; 32)$ & 0.26 & $(50 ; 40)$ & 0.49 & \begin{tabular}{|l|}
$(55.6 ; 33.4)$ \\
\end{tabular} & 0.54 & $(56 ; 26)$ & 0.67 \\
\hline & & $59.5 \pm 22.7$ & & $47.8 \pm 29$ & & $59 \pm 30$ & & $59.2 \pm 23.6$ & \\
\hline & Town/village & $(66 ; 30)$ & & \begin{tabular}{|l|}
$(60 ; 37.5)$ \\
\end{tabular} & & $(77.77 ; 41.7)$ & & \begin{tabular}{|l}
$(58.75 ; 30.13)$ \\
\end{tabular} & \\
\hline
\end{tabular}




\begin{tabular}{|c|c|c|c|c|c|c|c|c|c|}
\hline \multirow{3}{*}{\multicolumn{2}{|c|}{ Before treatment }} & \multicolumn{2}{|c|}{ Physical functioning } & \multicolumn{2}{|l|}{ Social functioning } & \multicolumn{2}{|l|}{ Physical role } & \multicolumn{2}{|l|}{ Emotional role } \\
\hline & & Avg. \pm SD & \multirow{2}{*}{$p$} & Avg. \pm SD & \multirow{2}{*}{ p } & Avg. \pm SD & \multirow{2}{*}{ p } & Avg. \pm SD & \multirow{2}{*}{$p$} \\
\hline & & M; IQR & & M; IQR & & M; IQR & & M; IQR & \\
\hline \multirow{2}{*}{ Gender } & \multirow{2}{*}{ Male } & $78.2 \pm 29$ & \multirow{2}{*}{0.005} & $83.1 \pm 23.7$ & \multirow{2}{*}{0.31} & $57.4 \pm 44.1$ & \multirow{2}{*}{0.06} & $44.9 \pm 33.1$ & \multirow{2}{*}{0.08} \\
\hline & & \begin{tabular}{|l|}
$(90 ; 30)$ \\
\end{tabular} & & $(100 ; 24.99)$ & & $(75 ; 100)$ & & $(50 ; 75)$ & \\
\hline \multirow{5}{*}{ Marriage status } & \multirow{2}{*}{ Married } & $54.5 \pm 33.3$ & \multirow{5}{*}{0.441} & $75.1 \pm 30.4$ & \multirow{5}{*}{0.19} & $31.1 \pm 43.1$ & \multirow{5}{*}{0.72} & $23 \pm 33$ & \multirow{5}{*}{0.44} \\
\hline & & $(75 ; 55)$ & & $(88.9 ; 33.33)$ & & $(50 ; 100)$ & & $(50 ; 75)$ & \\
\hline & Single & $76.3 \pm 21.4$ & & $85.2 \pm 22.4$ & & $39.6 \pm 43.2$ & & $29.2 \pm 35.1$ & \\
\hline & Singre & $(90 ; 40)$ & & $(88.9 ; 22.2)$ & & $(50 ; 100)$ & & $(25 ; 75)$ & \\
\hline & Widow/widower & $45 \pm 14.1$ & & $55.6 \pm 31.4$ & & $0 \pm 0$ & & $0 \pm 0$ & \\
\hline \multirow{7}{*}{ Education } & Literate & $(85 ; 60)$ & \multirow{7}{*}{0.01} & $(100 ; 33.3)$ & \multirow{7}{*}{0.3} & $(50 ; 100)$ & & $(50 ; 75)$ & \\
\hline & Flementary school & $72.8 \pm 29.4$ & & $83.5 \pm 24.6$ & & $52.4 \pm 46$ & 020 & $43.9 \pm 34.8$ & 0,53 \\
\hline & Elementary scnool & $(80 ; 47.5)$ & & \begin{tabular}{|l|}
$(100 ; 38.87)$ \\
\end{tabular} & & $(50 ; 100)$ & 0.02 & \begin{tabular}{|l|}
$(75 ; 75)$ \\
\end{tabular} & 0.53 \\
\hline & & $82.2 \pm 23.9$ & & $85 \pm 16.3$ & & $64.1 \pm 42.5$ & & $46.7 \pm 33.1$ & \\
\hline & High school & \begin{tabular}{|l|}
$(90 ; 30)$ \\
\end{tabular} & & $(88.88 ; 22.22)$ & & $(75 ; 100)$ & & $(75 ; 75)$ & \\
\hline & University & $79.4 \pm 27.4$ & & $75 \pm 25$ & & $50 \pm 53.5$ & & $28.1 \pm 33.9$ & \\
\hline & University & $(92.5 ; 41.25)$ & & $(83.34 ; 27.79)$ & & $(50 ; 100)$ & & $(12.5 ; 68.75)$ & \\
\hline & Provinge/city & $56.9 \pm 31.7$ & & $78.6 \pm 25.7$ & & $32.7 \pm 42.9$ & & $22.1 \pm 31.1$ & \\
\hline & Province/city & $(80 ; 40)$ & & \begin{tabular}{|l|}
$(88.9 ; 22.22)$ \\
\end{tabular} & & \begin{tabular}{|l|}
$(75 ; 100)$ \\
\end{tabular} & & \begin{tabular}{|l|}
$(25 ; 75)$ \\
\end{tabular} & \\
\hline Place of & District & $57.2 \pm 32.5$ & 0881 & $70.4 \pm 32.9$ & 085 & $22.2 \pm 38.4$ & 069 & $25 \pm 37.5$ & 077 \\
\hline residence & District & \begin{tabular}{|l|}
$(70 ; 55)$ \\
\end{tabular} & 0.881 & $(88.8 ; 44.4)$ & 0.85 & $(50 ; 100)$ & 0.69 & \begin{tabular}{|l|}
$(50 ; 75)$ \\
\end{tabular} & 0.11 \\
\hline
\end{tabular}




\begin{tabular}{|c|c|c|c|c|c|c|c|c|c|}
\hline \multicolumn{10}{|l|}{ Table 3. Continued } \\
\hline \multirow{3}{*}{\multicolumn{2}{|c|}{ Before treatment }} & \multicolumn{2}{|l|}{ Mental health } & \multicolumn{2}{|l|}{ Energy } & \multicolumn{2}{|l|}{ Bodily pain } & \multicolumn{2}{|l|}{ General health } \\
\hline & & \multirow{2}{*}{\begin{tabular}{|l|} 
Avg. \pm SD \\
M; IQR \\
\end{tabular}} & \multirow{2}{*}{$p$} & Avg. \pm SD & \multirow{2}{*}{$p$} & Avg. \pm SD & \multirow{2}{*}{$p$} & Avg. \pm SD & \multirow{2}{*}{$p$} \\
\hline & & & & M; IQR & & M; IQR & & M; IQR & \\
\hline \multicolumn{10}{|c|}{$6^{\text {th }}$ Month of treatment } \\
\hline \multirow{4}{*}{ Gender } & \multirow{2}{*}{ Male } & $58.1 \pm 22.5$ & \multirow{4}{*}{0.72} & $49.2 \pm 26.9$ & \multirow{4}{*}{0.35} & $64.2 \pm 26.8$ & \multirow{4}{*}{0.02} & $61.2 \pm 23.1$ & \multirow{4}{*}{0.14} \\
\hline & & $(60 ; 28)$ & & $(56 ; 36.25)$ & & \begin{tabular}{|l|}
$(66.66 ; 36.17)$ \\
\end{tabular} & & $(61 ; 32.5)$ & \\
\hline & \multirow{2}{*}{ Female } & $56.5 \pm 21.4$ & & $44.1 \pm 28.4$ & & $51.6 \pm 27.2$ & & $54.8 \pm 20.3$ & \\
\hline & & $(56 ; 26)$ & & $(47.5 ; 42.5)$ & & $(55.57 ; 41.7)$ & & \begin{tabular}{|l}
$(59.25 ; 23.25)$ \\
\end{tabular} & \\
\hline \multirow{6}{*}{ Marriage status } & \multirow{2}{*}{ Married } & $48.4 \pm 20.8$ & \multirow{6}{*}{0.93} & $34.7 \pm 29.8$ & \multirow{6}{*}{0.97} & $52 \pm 33.8$ & \multirow{6}{*}{0.04} & $55.4 \pm 24.4$ & \multirow{6}{*}{0.85} \\
\hline & & $(56 ; 24)$ & & $(50 ; 40)$ & & $(55.6 ; 33.4)$ & & $(61 ; 27.5)$ & \\
\hline & \multirow{2}{*}{ Single } & $58.3 \pm 20.7$ & & $40 \pm 22.3$ & & $60.2 \pm 28.2$ & & $58.3 \pm 28.5$ & \\
\hline & & $(64 ; 40)$ & & $(45 ; 45)$ & & $(66.7 ; 44.49)$ & & \begin{tabular}{|l}
$(57.5 ; 31)$ \\
\end{tabular} & \\
\hline & \multirow{2}{*}{ Widow/widower } & $52 \pm 5.7$ & & $25 \pm 35.4$ & & $22.2 \pm 15.7$ & & $67.3 \pm 12.4$ & \\
\hline & & $(60 ; 20)$ & & $(55 ; 58.75)$ & & \begin{tabular}{|l|}
$(27.76 ; 27.75)$ \\
\end{tabular} & & $(61 ; 20.75)$ & \\
\hline \multirow{10}{*}{ Education } & \multirow{2}{*}{ Illiterate } & $46.9 \pm 17.1$ & & $28.7 \pm 24.5$ & & $44.4 \pm 29.7$ & & $50.7 \pm 22.9$ & \\
\hline & & $(48 ; 16)$ & & $(30 ; 50)$ & & $(44.44 ; 44.45)$ & & $(62.5 ; 34)$ & \\
\hline & & $61.9 \pm 27.3$ & & $52.3 \pm 30.3$ & & $56.3 \pm 22.8$ & & $56.6 \pm 13.7$ & \\
\hline & Literate & $(68 ; 28)$ & & $(55 ; 35)$ & & \begin{tabular}{|l}
$(66.66 ; 44.67)$ \\
\end{tabular} & & \begin{tabular}{|l}
$(52.5 ; 17.5)$ \\
\end{tabular} & \\
\hline & & $58.7 \pm 22.3$ & & \begin{tabular}{|l|}
$49.1 \pm 28$ \\
\end{tabular} & & $60.7 \pm 28.1$ & & $58.6 \pm 23.5$ & \\
\hline & Elementary school & $(60 ; 28)$ & 0.26 & \begin{tabular}{|l|}
$(50 ; 40)$ \\
\end{tabular} & 0.06 & \begin{tabular}{|l}
$(55.6 ; 38.94)$ \\
\end{tabular} & 0.22 & \begin{tabular}{|l}
$(61 ; 31.75)$ \\
\end{tabular} & 0.56 \\
\hline & & $60.7 \pm 18.1$ & & $52.8 \pm 25.8$ & & $65.2 \pm 25.1$ & & $63.1 \pm 23.1$ & \\
\hline & High school & $(64 ; 24)$ & & $(50 ; 30)$ & & $(66.7 ; 33.36)$ & & $(61 ; 21.75)$ & \\
\hline & & $51.5 \pm 25$ & & $40.6 \pm 20.6$ & & $55.6 \pm 32.5$ & & $59.4 \pm 22.6$ & \\
\hline & University & $(58 ; 45)$ & & \begin{tabular}{|l}
$(37.5 ; 35)$ \\
\end{tabular} & & $(50 ; 63.90)$ & & $(56.25 ; 40.13)$ & \\
\hline & & $54.6 \pm 25$ & & $33.8 \pm 28.9$ & & $50.9 \pm 31.5$ & & $56.9 \pm 23.4$ & \\
\hline & Province/city & \begin{tabular}{|l|}
$(64 ; 24)$ \\
\end{tabular} & & \begin{tabular}{|l|}
$(50 ; 45)$ \\
\end{tabular} & & \begin{tabular}{|l|}
$(55.6 ; 33.4)$ \\
\end{tabular} & & \begin{tabular}{|l|}
$(62.5 ; 21.75)$ \\
\end{tabular} & \\
\hline Place of & Dictrict & $39.6 \pm 23.5$ & 04 & $35.6 \pm 21.9$ & 054 & $45.7 \pm 37.5$ & 055 & $46.3 \pm 22.8$ & 051 \\
\hline residence & District & $(52 ; 36)$ & 0.44 & $(50 ; 40)$ & 0.54 & $(55.6 ; 33.4)$ & 0.55 & $(57.5 ; 26)$ & 0.51 \\
\hline & & $51.3 \pm 22.2$ & & $38.4 \pm 30.8$ & & $59.7 \pm 32.2$ & & $61.7 \pm 27.9$ & \\
\hline & Town/village & \begin{tabular}{|l}
$(62 ; 28)$ \\
\end{tabular} & & $(50 ; 47.5)$ & & $\begin{array}{l}(61.13 ; 55.56) \\
\end{array}$ & & $(62.5 ; 33.88)$ & \\
\hline
\end{tabular}




\begin{tabular}{|c|c|c|c|c|c|c|c|c|c|}
\hline \multicolumn{10}{|l|}{ Before treatment } \\
\hline & & \multicolumn{2}{|c|}{ Physical functioning } & \multicolumn{2}{|c|}{ Social functioning } & \multicolumn{2}{|l|}{ Physical role } & \multicolumn{2}{|c|}{ Emotional role } \\
\hline \multirow{2}{*}{ Gender } & Male & $85.3 \pm 16.4$ & \multirow{2}{*}{0.00009} & $88.9 \pm 15.3$ & \multirow{2}{*}{0.03} & $68.8 \pm 41.3$ & \multirow{2}{*}{0.002} & $56.3 \pm 29.1$ & \multirow{2}{*}{0.012} \\
\hline & Female & $27.5 \pm 31.7$ & & $65.3 \pm 40$ & & $12.5 \pm 35.4$ & & $21.9 \pm 33.9$ & \\
\hline Marriage status & Single & $81.9 \pm 28$ & 0.202 & $72.2 \pm 29.1$ & 0.215 & $59.4 \pm 46.2$ & 0.641 & $40.6 \pm 37.6$ & 0.576 \\
\hline \multirow{4}{*}{ Education } & Illiterate & $17.5 \pm 3.5$ & \multirow{4}{*}{0.234} & $77.8 \pm 31.4$ & \multirow{4}{*}{0.946} & $50 \pm 70.7$ & \multirow{4}{*}{0.393} & $37.5 \pm 53$ & \multirow{4}{*}{0.389} \\
\hline & Literate & $62.5 \pm 10.6$ & & $94.4 \pm 7.9$ & & $0 \pm 0$ & & $12.5 \pm 17.7$ & \\
\hline & Elementary school & $72 \pm 36.3$ & & $81.5 \pm 27.4$ & & $48.3 \pm 50.4$ & & $45 \pm 35.6$ & \\
\hline & High school & $72.1 \pm 31.9$ & & $84.1 \pm 33.2$ & & $71.4 \pm 36.6$ & & $64.3 \pm 19.7$ & \\
\hline \multirow{6}{*}{ Occupation } & Employee & $88.3 \pm 16.1$ & \multirow{6}{*}{0.005} & $92.5 \pm 12.8$ & \multirow{6}{*}{0.196} & $83.3 \pm 28.9$ & & $75 \pm 0$ & \\
\hline & Housewife & $29.3 \pm 33.8$ & & $73 \pm 36.2$ & & $14.3 \pm 37.8$ & & $21.4 \pm 36.5$ & \\
\hline & Worker & $90 \pm 14.1$ & & $88.9 \pm 15.7$ & & $62.5 \pm 53$ & 0.113 & $37.5 \pm 53$ & 0.071 \\
\hline & Self-employment & $78.8 \pm 21.2$ & & $90.2 \pm 16.2$ & & $56.2 \pm 47.7$ & & $53.1 \pm 28.1$ & \\
\hline & Student & $50 \pm 49.5$ & & $38.9 \pm 39.3$ & & $25 \pm 35.4$ & & $12.5 \pm 17.7$ & \\
\hline & Other & $87.5 \pm 13.2$ & & $94.4 \pm 6.4$ & & $75 \pm 50$ & & $62.5 \pm 25$ & \\
\hline & Province/city & $74.6 \pm 29.1$ & & $66.7 \pm 34.3$ & & $77.8 \pm 18.4$ & & $36.1 \pm 48.6$ & \\
\hline Place of & District & $59.2 \pm 46.1$ & 0.656 & $68.8 \pm 34$ & 0.259 & $82.1 \pm 26.4$ & 0.449 & $52.7 \pm 46.8$ & 0.183 \\
\hline & Town/village & $74.6 \pm 29.1$ & & $90.6 \pm 25.6$ & & $59.6 \pm 45.1$ & & $57.7 \pm 29.6$ & \\
\hline & Illiterate & $27.5 \pm 38.9$ & & $94.4 \pm 7.9$ & & $0 \pm 0$ & & $0 \pm 0$ & \\
\hline & Literate & $85 \pm 21.2$ & & $94.4 \pm 7.9$ & & $0 \pm 0$ & & $0 \pm 0$ & \\
\hline Education & Elementary school & $85 \pm 27.7$ & 0.15 & $83 \pm 23$ & 0.52 & $66.7 \pm 43$ & 0.101 & $56.7 \pm 32$ & 0.085 \\
\hline & High school & $75.7 \pm 30.5$ & & $74.6 \pm 43.9$ & & $60.7 \pm 49.7$ & & $46.4 \pm 36.6$ & \\
\hline & University & $77.5 \pm 10.6$ & & $50 \pm 39.3$ & & $25 \pm 35.4$ & & $37.5 \pm 53$ & \\
\hline & Unemployed & $100 \pm 0$ & & $77.8 \pm 31.4$ & & $87.5 \pm 17.7$ & & $75 \pm 0$ & \\
\hline & Employee & $81.7 \pm 12.6$ & & $74.1 \pm 44.9$ & & $66.7 \pm 57.7$ & & $75 \pm 0$ & \\
\hline & Housewife & $50.7 \pm 38$ & & $84.1 \pm 23.9$ & & $3.6 \pm 9.4$ & & $21.4 \pm 36.6$ & \\
\hline Occupation & Worker & $100 \pm 0$ & 0.051 & $88.9 \pm 15.7$ & 0.881 & $100 \pm 0$ & 0.025 & $75 \pm 0$ & 0.105 \\
\hline & Self-employment & $88.8 \pm 18.1$ & & $83.3 \pm 35.6$ & & $65.6 \pm 48.1$ & & $50 \pm 43.3$ & \\
\hline & Student & $52.5 \pm 46$ & & $50 \pm 39.3$ & & $37.5 \pm 17.7$ & & $10.7 \pm 28.3$ & \\
\hline & Other & $92.5 \pm 15$ & & $83.3 \pm 26.4$ & & $68.8 \pm 47.3$ & & $75 \pm 0$ & \\
\hline & Province/city & $83.1 \pm 51.6$ & & $80.3 \pm 33.7$ & & $61.5 \pm 45.2$ & & $53.8 \pm 33.6$ & \\
\hline $\begin{array}{l}\text { rlace ot } \\
\text { residence }\end{array}$ & District & $72.5 \pm 47.1$ & 0.72 & $77.8 \pm 27.2$ & 0.974 & $45.8 \pm 51$ & 0.657 & $37.5 \pm 41.1$ & 0.473 \\
\hline & Town/village & $74.4 \pm 33.2$ & & $81.5 \pm 27.8$ & & $44.4 \pm 48.1$ & & $36.1 \pm 37.7$ & \\
\hline
\end{tabular}




\begin{tabular}{|c|c|c|c|c|c|c|c|c|c|}
\hline \multicolumn{10}{|l|}{ Before treatment } \\
\hline & & \multicolumn{2}{|c|}{ Mental health } & \multicolumn{2}{|l|}{ Energy } & \multicolumn{2}{|l|}{ Bodily pain } & \multicolumn{2}{|c|}{ General health } \\
\hline \multirow{2}{*}{ Gender } & Male & $67.8 \pm 20.4$ & \multirow{2}{*}{0.088} & $51.3 \pm 33.9$ & \multirow{2}{*}{0.014} & $76.1 \pm 27$ & \multirow{2}{*}{0.011} & $53 \pm 21$ & \multirow{2}{*}{0.023} \\
\hline & Female & $50.5 \pm 29.9$ & & $17.5 \pm 19.8$ & & $41.7 \pm 37$ & & $32.3 \pm 19.1$ & \\
\hline Marriage status & Single & $68 \pm 30.4$ & 0.488 & $60.6 \pm 33.4$ & 0.059 & $69.4 \pm 34$ & 0.757 & $45.7 \pm 24.4$ & 0.842 \\
\hline \multirow{4}{*}{ Education } & Illiterate & $48 \pm 5.65$ & \multirow{4}{*}{0.282} & $35 \pm 35.4$ & \multirow{4}{*}{0.531} & $16.7 \pm 7.9$ & \multirow{4}{*}{0.272} & $18.8 \pm 1.76$ & \multirow{4}{*}{0.222} \\
\hline & Literate & $40 \pm 11.3$ & & $20 \pm 0$ & & $55.6 \pm 31.4$ & & $43 \pm 11.3$ & \\
\hline & Elementary school & $69.9 \pm 20.7$ & & $44.7 \pm 35.3$ & & $70.4 \pm 32.7$ & & $54.7 \pm 21.8$ & \\
\hline & High school & $54.9 \pm 33.3$ & & $33.6 \pm 37.7$ & & $71.4 \pm 35.1$ & & $40.1 \pm 25.1$ & \\
\hline \multirow{6}{*}{ Occupation } & Employee & $74.7 \pm 6.1$ & \multirow{6}{*}{0.234} & $83.3 \pm 15.3$ & \multirow{6}{*}{0.611} & $92.6 \pm 12.8$ & & $35.8 \pm 17.6$ & \\
\hline & Housewife & $56.6 \pm 26.4$ & & $20 \pm 20$ & & $44.4 \pm 39$ & & $46.8 \pm 13.1$ & \\
\hline & Worker & $84 \pm 22.6$ & & $62.5 \pm 17.7$ & & $55.6 \pm 15.7$ & 0.234 & $49.1 \pm 22.1$ & 0.232 \\
\hline & Self-employment & $61.5 \pm 24.7$ & & $28.8 \pm 30.6$ & & $73.6 \pm 32.5$ & & $28 \pm 29$ & \\
\hline & Student & $40 \pm 45.3$ & & $35 \pm 49.5$ & & $38.9 \pm 23.6$ & & $70.1 \pm 28.8$ & \\
\hline & Other & $64 \pm 23.8$ & & $65 \pm 34.2$ & & $80.6 \pm 31.9$ & & $41 \pm 14.1$ & \\
\hline & Province/city & $30.6 \pm 34.9$ & & $68.9 \pm 20.5$ & & $32.8 \pm 29.7$ & & $50.6 \pm 33.4$ & \\
\hline Place of & District & $46.4 \pm 33.8$ & 0.393 & $62.9 \pm 24.2$ & 0.649 & $42.6 \pm 33.9$ & 0.237 & $66.3 \pm 33.5$ & 0.602 \\
\hline & Town/village & $64 \pm 25.5$ & & $46.5 \pm 32.4$ & & $72.6 \pm 31.6$ & & $51.6 \pm 50.6$ & \\
\hline & Illiterate & $60 \pm 5.7$ & & $40 \pm 14.1$ & & $33.3 \pm 15.7$ & & $41.3 \pm 1.8$ & \\
\hline & Literate & $64 \pm 17$ & & $35 \pm 21.2$ & & $72.2 \pm 39.3$ & & $62.5 \pm 17.7$ & \\
\hline Education & Elementary school & $68 \pm 19.8$ & 0.462 & $54.7 \pm 27.7$ & 0.604 & $71.9 \pm 30.5$ & 0.479 & $62.2 \pm 22.8$ & 0.603 \\
\hline & High school & $49.7 \pm 36$ & & $36.4 \pm 38.4$ & & $65.1 \pm 26.8$ & & $47.7 \pm 27$ & \\
\hline & University & $42 \pm 31.1$ & & $30 \pm 35.4$ & & $50 \pm 39.3$ & & $49.8 \pm 40.7$ & \\
\hline & Unemployed & $72 \pm 11.3$ & & $75 \pm 7.1$ & & $88.9 \pm 15.7$ & & $46 \pm 21.2$ & \\
\hline & Employee & $53.3 \pm 28.9$ & & $45 \pm 35$ & & $59.3 \pm 39$ & & $57.7 \pm 38.9$ & \\
\hline & Housewife & $60.6 \pm 15$ & & $35.7 \pm 23$ & & $54 \pm 38.2$ & & $53.5 \pm 21.9$ & \\
\hline Occupation & Worker & $76 \pm 33.9$ & 0.862 & $50 \pm 28.3$ & 0.73 & $77.8 \pm 31.4$ & 0.716 & $58 \pm 2.8$ & 0.974 \\
\hline & Self-employment & $60.5 \pm 34$ & & $45 \pm 38.5$ & & $68.1 \pm 22.6$ & & $61.3 \pm 26.1$ & \\
\hline & Student & $40 \pm 33.9$ & & $30 \pm 35.4$ & & $50 \pm 39.3$ & & $44.3 \pm 48.4$ & \\
\hline & Other & $64 \pm 22.9$ & & $57.5 \pm 28.7$ & & $77.8 \pm 27.2$ & & $59.9 \pm 17.9$ & \\
\hline & Province/city & $64.6 \pm 27.4$ & & $46.9 \pm 29.1$ & & $70.9 \pm 25.5$ & & $62 \pm 23.5$ & \\
\hline $\begin{array}{l}\text { Place ot } \\
\text { residence }\end{array}$ & District & $48.7 \pm 25$ & 0.425 & $45 \pm 32.7$ & 0.987 & $70.4 \pm 34.2$ & 0.467 & $48.4 \pm 27.2$ & 0.474 \\
\hline & Town/village & $63.1 \pm 21.5$ & & $45 \pm 32.8$ & & $55.6 \pm 33.3$ & & $53.1 \pm 22.6$ & \\
\hline
\end{tabular}




\begin{tabular}{|c|c|c|c|c|c|c|c|c|c|}
\hline \multicolumn{10}{|l|}{ Before treatment } \\
\hline & & \multicolumn{2}{|c|}{ Physical functioning } & \multicolumn{2}{|c|}{ Social functioning } & \multicolumn{2}{|l|}{ Physical role } & \multicolumn{2}{|c|}{ Emotional role } \\
\hline \multicolumn{10}{|c|}{$6^{\text {th }}$ month of treatment } \\
\hline Gender & Male & $75.3 \pm 28.1$ & 0.112 & $80.6 \pm 26$ & 0.271 & $48.8 \pm 44.8$ & 0.106 & $36.3 \pm 36.7$ & 0.258 \\
\hline \multirow{2}{*}{ Marriage status } & Married & $61.3 \pm 26.8$ & \multirow{2}{*}{0.062} & $84.4 \pm 82.5$ & \multirow{2}{*}{0.806} & $40 \pm 81.9$ & \multirow{2}{*}{0.974} & $28.8 \pm 40.6$ & \multirow{2}{*}{0.575} \\
\hline & Single & $82.5 \pm 61.3$ & & $81.9 \pm 84.4$ & & $40.6 \pm 40$ & & $37.5 \pm 28.8$ & \\
\hline \multirow{2}{*}{ Education } & Illiterate & $37.5 \pm 37.5$ & \multirow{2}{*}{0.325} & $83.3 \pm 83.3$ & \multirow{2}{*}{0.369} & $25 \pm 25$ & \multirow{2}{*}{0.371} & $0 \pm 0$ & \multirow{2}{*}{0.152} \\
\hline & Literate & $42.5 \pm 42.5$ & & $88.9 \pm 88.9$ & & $0 \pm 0$ & & $0 \pm 0$ & \\
\hline \multirow{7}{*}{ Occupation } & Unemployed & $90 \pm 14.1$ & \multirow{7}{*}{0.539} & $100 \pm 0$ & \multirow{7}{*}{0.624} & $100 \pm 0$ & \multirow{7}{*}{0.216} & $75 \pm 0$ & \multirow{7}{*}{0.215} \\
\hline & Employee & $76.7 \pm 22.5$ & & $74.1 \pm 44.9$ & & $58.3 \pm 52$ & & $33.3 \pm 38.2$ & \\
\hline & Housewife & $49.3 \pm 10.6$ & & $95.2 \pm 12.6$ & & $21.4 \pm 39.3$ & & $21.4 \pm 36.6$ & \\
\hline & Worker & $65 \pm 35.4$ & & $66.7 \pm 47.1$ & & $12.5 \pm 17.7$ & & $0 \pm 0$ & \\
\hline & Self-employment & $73.1 \pm 33.4$ & & $81.9 \pm 24.4$ & & $46.9 \pm 47.1$ & & $31.3 \pm 37.2$ & \\
\hline & Student & $62.5 \pm 38.9$ & & $72.2 \pm 7.9$ & & $0 \pm 0$ & & $0 \pm 0$ & \\
\hline & Other & $72.5 \pm 35.7$ & & $80.6 \pm 19$ & & $50 \pm 45.6$ & & $56.3 \pm 37.5$ & \\
\hline $\begin{array}{l}\text { Place of } \\
\text { residence }\end{array}$ & Province/city & $72.3 \pm 24.6$ & 0.492 & $87.2 \pm 16.3$ & 0.731 & $44.2 \pm 45.8$ & 0.886 & $32.7 \pm 35.9$ & 0.806 \\
\hline
\end{tabular}




\begin{tabular}{|c|c|c|c|c|c|c|c|c|c|}
\hline \multicolumn{10}{|l|}{ Before treatment } \\
\hline & & \multicolumn{2}{|c|}{ Mental health } & \multicolumn{2}{|l|}{ Energy } & \multicolumn{2}{|l|}{ Bodily pain } & \multicolumn{2}{|c|}{ General health } \\
\hline & & Avg. \pm SD & $p$ & Avg. \pm SD & p & Avg. \pm SD & $\mathrm{p}$ & Avg. \pm SD & $\mathbf{p}$ \\
\hline \multicolumn{10}{|c|}{$6^{\text {th }}$ month of treatment } \\
\hline \multirow{2}{*}{ Gender } & Male & $57.2 \pm 22.7$ & \multirow{2}{*}{0.64} & $44.8 \pm 27.3$ & \multirow{2}{*}{0.121} & $58.3 \pm 33$ & \multirow{2}{*}{0.309} & $62.1 \pm 25.1$ & \multirow{2}{*}{0.113} \\
\hline & Female & $53 \pm 16.7$ & & $26.3 \pm 28.3$ & & $44.4 \pm 29.1$ & & $44.8 \pm 25.8$ & \\
\hline \multirow{2}{*}{ Marriage status } & Married & $57 \pm 37.5$ & \multirow{2}{*}{0.697} & $37.8 \pm 53.5$ & \multirow{2}{*}{0.622} & $53.3 \pm 43.8$ & \multirow{2}{*}{0.793} & $56.8 \pm 56.9$ & \multirow{2}{*}{0.894} \\
\hline & Single & $53.5 \pm 57$ & & $43.8 \pm 37.8$ & & $56.9 \pm 53.3$ & & $58.3 \pm 56.8$ & \\
\hline \multirow{5}{*}{ Education } & Illiterate & $48 \pm 0$ & \multirow{5}{*}{0.463} & $15 \pm 21.2$ & \multirow{5}{*}{0.561} & $50 \pm 7.9$ & \multirow{5}{*}{0.78} & $41.3 \pm 37.1$ & \multirow{5}{*}{0.66} \\
\hline & Literate & $36 \pm 39.6$ & & $20 \pm 28.3$ & & $27.8 \pm 7.9$ & & $45.5 \pm 18.4$ & \\
\hline & Elementary school & $62.1 \pm 18.9$ & & $45.7 \pm 24.6$ & & $58.5 \pm 35$ & & $62.9 \pm 24.6$ & \\
\hline & High school & $52.6 \pm 23$ & & $38.6 \pm 35.8$ & & $57.1 \pm 35.4$ & & $57.2 \pm 25.8$ & \\
\hline & University & $50 \pm 19.8$ & & $40 \pm 42.4$ & & $44.4 \pm 31.4$ & & $41.8 \pm 48.4$ & \\
\hline \multirow{7}{*}{ Occupation } & Unemployed & $70 \pm 8.5$ & \multirow{7}{*}{0.862} & $70 \pm 0$ & \multirow{7}{*}{0.713} & $100 \pm 0$ & \multirow{7}{*}{0.425} & $57.5 \pm 47.5$ & \multirow{7}{*}{0.924} \\
\hline & Employee & $61.3 \pm 22$ & & $40 \pm 30$ & & $55.6 \pm 29.4$ & & $60 \pm 47.5$ & \\
\hline & Housewife & $58.3 \pm 8$ & & $30 \pm 28.3$ & & $46 \pm 31.1$ & & $48.7 \pm 25.1$ & \\
\hline & Worker & $56 \pm 50.9$ & & $25 \pm 35.4$ & & $66.7 \pm 31.4$ & & $52.5 \pm 28.3$ & \\
\hline & Self-employment & $59 \pm 18.6$ & & $44.4 \pm 29.5$ & & $43.1 \pm 37.8$ & & $66.5 \pm 21.5$ & \\
\hline & Student & $40 \pm 33.9$ & & $35 \pm 49.5$ & & $50 \pm 23.6$ & & $46.8 \pm 41.4$ & \\
\hline & Other & $43 \pm 28.5$ & & $40 \pm 27.1$ & & $63.9 \pm 27.8$ & & $58.6 \pm 21.1$ & \\
\hline \multirow{3}{*}{$\begin{array}{l}\text { Place of } \\
\text { residence }\end{array}$} & Province/city & $63.4 \pm 18.1$ & \multirow{3}{*}{0.111} & $48.5 \pm 27.9$ & \multirow{3}{*}{0.3} & $58.1 \pm 31.8$ & \multirow{3}{*}{0.823} & $65 \pm 22.1$ & \multirow{3}{*}{0.243} \\
\hline & District & $42 \pm 16.3$ & & $33.3 \pm 26.6$ & & $48.1 \pm 34.2$ & & $43.5 \pm 23.7$ & \\
\hline & Town/village & $54.7 \pm 24.1$ & & $30.6 \pm 29.2$ & & $53.1 \pm 34.1$ & & $55.1 \pm 31$ & \\
\hline
\end{tabular}




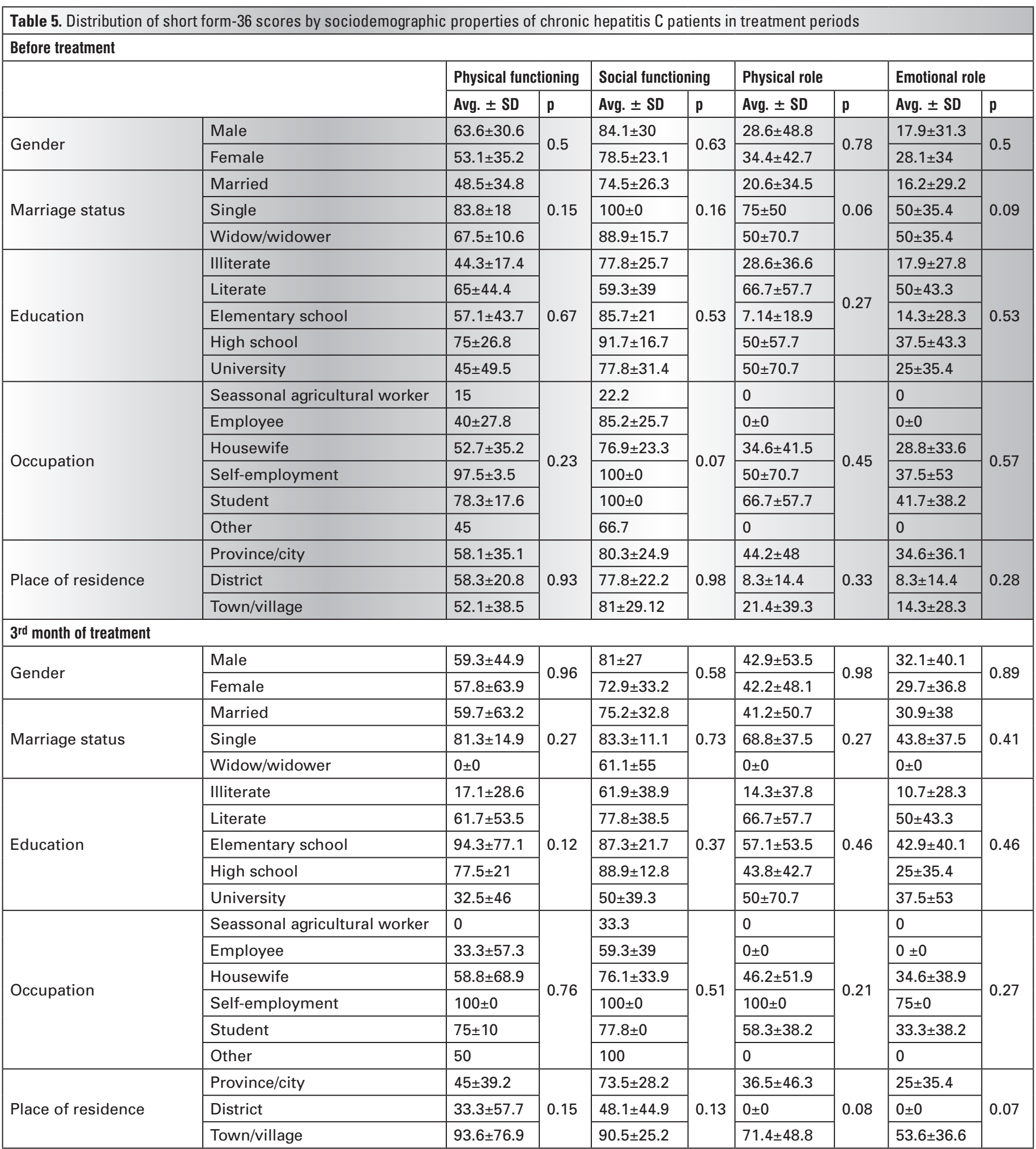




\begin{tabular}{|c|c|c|c|c|c|c|c|c|c|}
\hline \multicolumn{10}{|l|}{ Table 5. Continued } \\
\hline \multicolumn{10}{|l|}{ Before treatment } \\
\hline & & \multicolumn{2}{|c|}{ Mental health } & \multicolumn{2}{|l|}{ Energy } & \multicolumn{2}{|l|}{ Bodily pain } & \multicolumn{2}{|c|}{ General health } \\
\hline \multirow{2}{*}{ Gender } & Male & $65.7 \pm 21.8$ & \multirow{2}{*}{0.165} & $42.9 \pm 26.9$ & \multirow{2}{*}{0.39} & $60.3 \pm 29.3$ & \multirow{2}{*}{0.32} & $53 \pm 20.3$ & \multirow{2}{*}{0.13} \\
\hline & Female & $51.8 \pm 21.3$ & & $33.1 \pm 23.2$ & & $57.2 \pm 27.7$ & & $39.5 \pm 18.4$ & \\
\hline Marriage status & Widow/widower & $68 \pm 0$ & 0.132 & $70 \pm 14.1$ & 0.005 & $55.6 \pm 31.4$ & 0.03 & $48.8 \pm 15.9$ & 0.2 \\
\hline \multirow[b]{3}{*}{ Education } & Illiterate & $45.7 \pm 19.6$ & \multirow[b]{3}{*}{0.496} & $30 \pm 28.1$ & \multirow[b]{3}{*}{0.38} & $42.9 \pm 20.7$ & \multirow[b]{3}{*}{0.53} & $39.6 \pm 20.9$ & \multirow[b]{3}{*}{0.74} \\
\hline & Literate & $60 \pm 24$ & & $45 \pm 31.2$ & & $66.7 \pm 22.2$ & & $39.5 \pm 23.4$ & \\
\hline & Elementary school & $54.9 \pm 24.2$ & & $25.7 \pm 16.2$ & & $42.9 \pm 28.3$ & & $40.6 \pm 21.7$ & \\
\hline \multirow{5}{*}{ Occupation } & Employee & $62.7 \pm 27.2$ & \multirow{5}{*}{0.346} & $23.3 \pm 5.8$ & & $51.9 \pm 23.1$ & & $56.7 \pm 12.6$ & \\
\hline & Housewife & $50.5 \pm 21.5$ & & $32.7 \pm 25.8$ & & $43.6 \pm 26.2$ & & $36.9 \pm 18.2$ & \\
\hline & Self-employment & $50 \pm 19.8$ & & $65 \pm 21.2$ & 0.3 & $72.2 \pm 39.3$ & 0.21 & $66.8 \pm 4.6$ & 0.08 \\
\hline & Student & $76 \pm 14.4$ & & $48.3 \pm 18.9$ & & $77.8 \pm 22.2$ & & $54.8 \pm 17.7$ & \\
\hline & Other & 80 & & 50 & & 11.1 & & 42.5 & \\
\hline & Province/city & $56.6 \pm 23.1$ & & $38.8 \pm 27.2$ & & $58.1 \pm 32.4$ & & $47.2 \pm 19.3$ & \\
\hline Place of residence & \begin{tabular}{|l|} 
District \\
\end{tabular} & $66.7 \pm 6.1$ & 0.572 & $30 \pm 26.5$ & 0.82 & $44.4 \pm 11.1$ & 0.42 & $42.5 \pm 25.4$ & 0.58 \\
\hline & Town/village & $50.3 \pm 24.2$ & & $33.6 \pm 19.7$ & & $41.3 \pm 22.9$ & & $37.3 \pm 19.4$ & \\
\hline $3^{\text {rd }}$ month of treatment & & & & & & & & & \\
\hline Gender & Male & $41.7 \pm 26.2$ & 0.15 & $36.4 \pm 27.2$ & 088 & $73 \pm 27.1$ & 004 & $55.1 \pm 28.6$ & 08 \\
\hline Education & Elementary school & $50.3 \pm 24.5$ & 1 & $41.4 \pm 27.3$ & 0.13 & $60.3 \pm 26.3$ & 0.16 & $61.6 \pm 24$ & 0.45 \\
\hline & High school & $56 \pm 25.3$ & & $57.5 \pm 20.6$ & & \begin{tabular}{|l|l|}
$77.8 \pm 24$ \\
\end{tabular} & & $63.3 \pm 19.7$ & \\
\hline & University & $52 \pm 28.3$ & & $22.5 \pm 31.8$ & & $66.7 \pm 31.4$ & & $44.3 \pm 8.1$ & \\
\hline & Seassonal agricultural worker & 20 & & 0 & & 44.4 & & 22.5 & \\
\hline & Employee & $25.3 \pm 8.3$ & & $13.3 \pm 23.1$ & & $63 \pm 32.1$ & & $41.2 \pm 30.1$ & \\
\hline & Housewife & $58.5 \pm 22$ & 006 & $33.1 \pm 31.2$ & 027 & $45.3 \pm 25.8$ & 32 & $53.6 \pm 25.8$ & 038 \\
\hline Occupation & Self-employment & $66 \pm 19.8$ & 0.06 & $65 \pm 7.1$ & 0.21 & $66.7 \pm 31.4$ & 0.32 & $68.5 \pm 7.1$ & 0.38 \\
\hline & Student & $64 \pm 13.9$ & & $55 \pm 21.8$ & & $70.4 \pm 23.1$ & & $49.5 \pm 1.8$ & \\
\hline & Other & 24 & & 40 & & 100 & & 91 & \\
\hline & Province/city & $52.6 \pm 23.5$ & & $28.8 \pm 31.5$ & & $52.1 \pm 25$ & & $47.9 \pm 22.9$ & \\
\hline Place of residence & District & $45.3 \pm 26.6$ & 0.85 & $23.3 \pm 20.8$ & 0.21 & $48.1 \pm 44.9$ & 0.63 & $42.5 \pm 26$ & 0.18 \\
\hline & Town/village & $54.9 \pm 25.1$ & & $51.4 \pm 25.4$ & & $63.5 \pm 27$ & & $67.1 \pm 24.1$ & \\
\hline
\end{tabular}




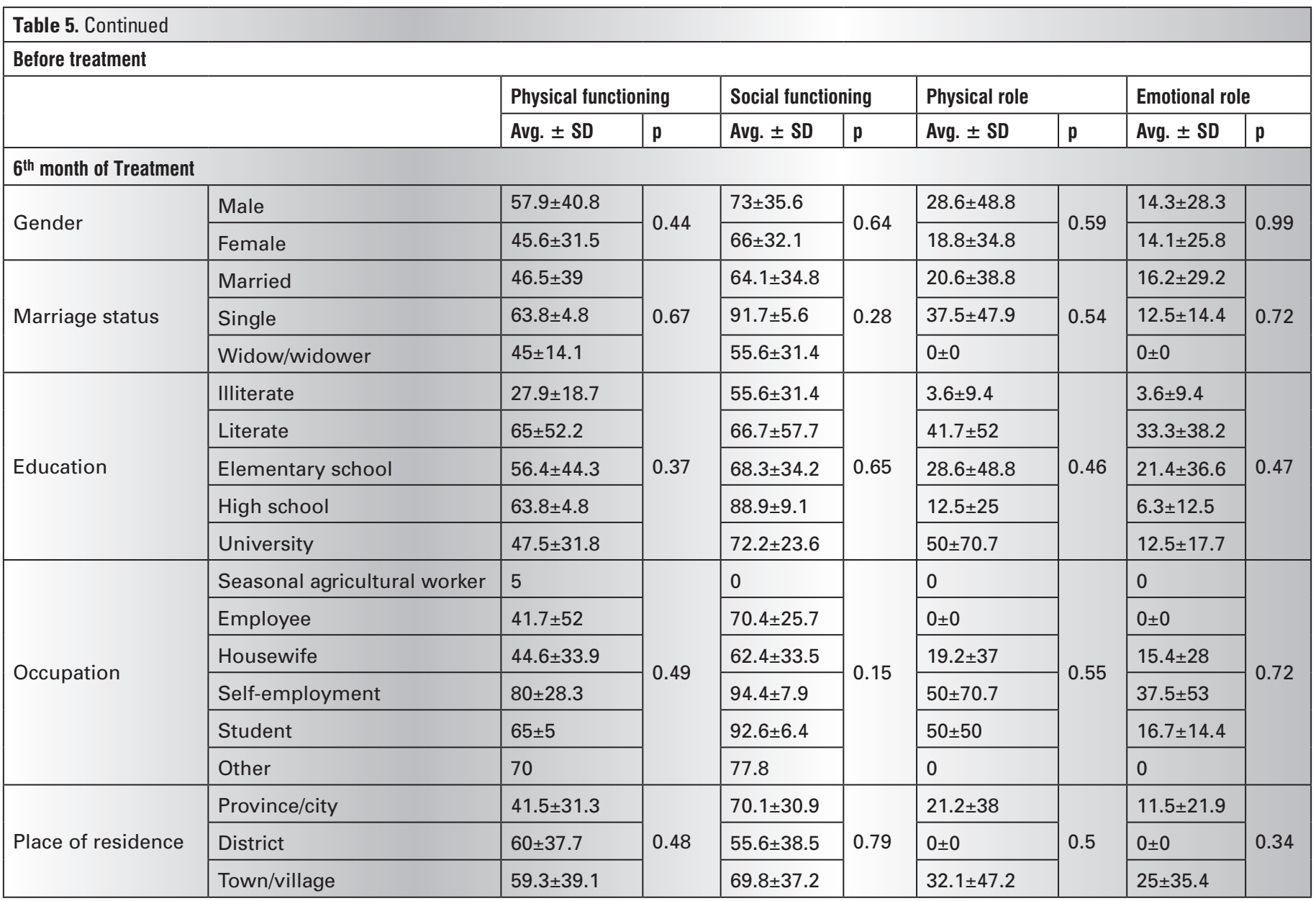

study, we also determined, in agreement with previous studies, lower HRQoL scores in patients with $\mathrm{CHC}$ compared to healthy controls. We observed statistically significant differences in physical functioning, physical role limitations, energy/vitality and general health scores in these patients compared to controls. Pojoga et al. (27) reported that patients with $\mathrm{CHB}$ had better general health, social functioning and mental health scores than patients with $\mathrm{CHC}$. Another study showed a significant decrease in $\mathrm{HRQOL}$ scores in patients with hepatitis $\mathrm{C}$ compared to that in patients with CHB (12). In our study, although HRQoL scores in patients with $\mathrm{CHC}$ were lower than those in patients with $\mathrm{CHB}$, the difference was only statistically significant in emotional role limitations scores. Lower HRQoL scores observed in patients with $\mathrm{CHC}$ compared to those with $\mathrm{CHB}$ may be attributed to symptoms such as lethargy and fatigue being more pronounced in the former and to this then affecting their emotional scores. When we compared the HORoL scores of patients with $\mathrm{CHC}$ during the treatment period with those of the control group, we determined a significant difference. This difference consisted of significantly low physical functioning, physical role limitations, emotional role limitations, mental health and vitality/energy scores in patients with hepatitis $\mathrm{C}$ at the $3^{\text {rd }}$ month of treatment and also a significantly lower social functioning score in addition to the other parameters at the $6^{\text {th }}$. HRQoL in chronic hepatitis is adversely affected during treatment. This may be due to drug side-effects such as fatigue, flu-like findings, such as myalgia, and changes in psychological state, concentration impairment and loss of libido adversely impacting patients' energy and social functioning $(28,29)$. Marcellin et al. $(30)$ investigated HRQoL in patients with $\mathrm{CHB}$ and $\mathrm{CHC}$ receiving PEG-IFN $\alpha-2 \mathrm{a}$ therapy and reported better HRQoL scores, particularly in the physical component, in patients with hepatitis B compared to those with hepatitis C. In our study, HRQoL scores during treatment were better in patients with $\mathrm{CHB}$ than in those with $\mathrm{CHC}$. The decrease in physical functioning and social functioning scores in patients with $\mathrm{CHC}$ at the $6^{\text {th }}$ month was statistically significant. HRQoL during treatment being lower in patients with $\mathrm{CHC}$ than in patients with $\mathrm{CHB}$ may be associated with the side-effects of combined IFN and ribavirin therapy. We also determined a significant decrease in $\mathrm{HRQOL}$ scores during treatment in our patients with $\mathrm{CHB}$ compared with the control group. This decrease was statistically significant at the $6^{\text {th }}$ month in physical, physical role limitations, emotional role limitations and energy/vitality scores.

\section{Conclusion}

Chronic viral hepatitis is a social health problem in Turkey. Chronic diseases can adversely affect quality of life in various ways. Patients with chronic hepatitis are exposed not only to the chronic effects of the disease, but also to undesirable effects of 


\begin{tabular}{|c|c|c|c|c|c|c|c|c|c|}
\hline \multicolumn{10}{|l|}{ Table 5. Continued } \\
\hline \multicolumn{10}{|l|}{ Before treatment } \\
\hline & & \multicolumn{2}{|l|}{ Mental health } & \multicolumn{2}{|l|}{ Energy } & \multicolumn{2}{|l|}{ Bodily pain } & \multicolumn{2}{|c|}{ General health } \\
\hline \multicolumn{10}{|l|}{$6^{\text {th }}$ month of Treatment } \\
\hline Gender & Male & $41.7 \pm 23.4$ & 0.63 & $30 \pm 25.2$ & 0.92 & $69.8 \pm 32.5$ & 0.07 & $56 \pm 28.7$ & 0.97 \\
\hline \multirow{3}{*}{ Marriage status } & Married & $38.4 \pm 17.3$ & \multirow{3}{*}{0.008} & $31.2 \pm 30.4$ & \multirow{3}{*}{0.95} & $50.3 \pm 34.9$ & \multirow{3}{*}{0.33} & $53.7 \pm 26.7$ & \multirow{3}{*}{0.75} \\
\hline & Single & $68 \pm 3.3$ & & $32.5 \pm 10.4$ & & $66.7 \pm 28.7$ & & $58.4 \pm 14.8$ & \\
\hline & Widow/widower & $52 \pm 5.7$ & & $25 \pm 35.4$ & & $22.2 \pm 15.7$ & & $67.3 \pm 12.4$ & \\
\hline \multirow{2}{*}{ Education } & Illiterate & $40 \pm 12$ & \multirow{2}{*}{0.31} & $14.3 \pm 19.9$ & \multirow{2}{*}{0.45} & $34.9 \pm 36$ & \multirow{2}{*}{0.66} & $48.9 \pm 28.1$ & \multirow{2}{*}{0.7} \\
\hline & University & $48 \pm 33.9$ & & $30 \pm 0$ & & $55.6 \pm 62.9$ & & $56.3 \pm 8.8$ & \\
\hline \multirow{6}{*}{ Occupation } & Seassonal agricultural worker & 12 & \multirow{6}{*}{0.004} & 0 & \multirow{6}{*}{0.74} & 22.2 & \multirow{6}{*}{0.61} & 27.5 & \multirow{6}{*}{0.45} \\
\hline & Employee & $22.7 \pm 6.1$ & & $23.3 \pm 20.8$ & & $51.9 \pm 44.9$ & & $49.2 \pm 32.1$ & \\
\hline & Housewife & $44.3 \pm 15.6$ & & $30 \pm 32.1$ & & $44.4 \pm 33.6$ & & $54.1 \pm 24$ & \\
\hline & Self-employment & $58 \pm 8.5$ & & $40 \pm 28.3$ & & $61.1 \pm 23.6$ & & $66.8 \pm 9.5$ & \\
\hline & Student & $69.3 \pm 2.3$ & & $36.7 \pm 7.6$ & & $63 \pm 33.9$ & & $57.8 \pm 18.1$ & \\
\hline & Other & 48 & & 60 & & 100 & & 96 & \\
\hline Place of residence & Province/city & $45.8 \pm 18.6$ & 0.63 & $19.2 \pm 22.3$ & 0.05 & $43.6 \pm 30.6$ & 0.26 & $48.7 \pm 22.5$ & 0.15 \\
\hline
\end{tabular}

IFN treatment and antiviral drugs. Measuring and evaluating quality of life is even more important in this patient group, in which severe decreases may be anticipated. Patients' quality of life is adversely affected by side-effects of treatment. This discomfort caused by treatment may also impair patients' compliance and willingness to continue with therapy. Emotional changes during treatment must be monitored and treatment should be provided when required. Considering the changes occurring in emotional and psychological states, psychiatric evaluation at least once during follow-up may be useful. Provision of counseling and guidance services can improve quality of life of patients with chronic viral hepatitis. Good standardization of HRQOL measures and application to patients with chronic diseases will identify negativities emerging and perhaps also be of assistance in coping with them.

\section{Ethics}

Ethic Committee Approval: The study was approved by Ataturk University Faculty of Medicine Ethics Committee (approval number: 65/2008).

Informed Consent: Informed consent was received from all.

Peer-review: Externally peer-reviewed.

\section{Authorship Contributions}

Surgical and Medical Practices: H.A., K.Ö., E.P., Concept: N.Ç., S.E., M.P., Design: S.E., H.A., K.Ö., E.P., Data Collection or
Processing: H.A., E.P., Analysis or Interpretation: H.A., K.Ö., M.P., S.E., Literature Search: H.A., K.Ö., E.P., Writing: H.A., K.Ö.

Conflict of Interest: No conflict of interest was declared by the authors.

Financial Disclosure: The authors declared that this study received no financial support.

\section{References}

1. Di Bisceglie AM. Hepatitis C and hepatocellular carcinoma. Hepatology. 1997;26(3 Suppl 1):34-38.

2. Özdemir D, Kurt H. Hepatit B virusu Infeksiyonlarının epidemiyolojisi. Tabak F, Balık I, Tekeli E, eds. Viral Hepatit 2007.Istanbul, Viral Hepatitle Savaşım Derneği, Oban Matbaası. 2007; 108-117.

3. Quer J, Esteban J. Epidemiology. In: Thomas HC, Lemon S, Zuckerman AJ eds. Viral hepatitis. Massachusetts, USA. Third Edition. Blackwell Publishing, 2005;407-425.

4. Çaylan R. Kronik hepatitler ve yaşam kalitesi. Tabak F, Balık I, Tekeli E, eds. Viral Hepatit. Istanbul Viral Hepatitle Savaşım Derneği, Oban Matbaası. 2007;376-384.

5. Ware JE Jr, Bayliss MS, Mannocchia M, Davis GL. Healthrelated quality of life in chronic hepatitis $\mathrm{C}$ : impact of disease and treatment response. The Interventional Therapy Group. Hepatology. 1999;30:550-555.

6. Mathew A, Peiffer LP, Rhoades K, McGarrity TJ. Improvement in quality of life measures in patients with refractory hepatitis $\mathrm{C}$, 
responding to re-treatment with Pegylated interferon alpha $-2 b$ and ribavirin. Health Qual Life Outcomes. 2006:4:30.

7. Ware JE Jr, Sherbourne CD. The MOS 36-item short-form health survey (SF-36). I. Conceptual framework and item selection. Med Care. 1992;30:473-483.

8. Koçyiğit H, Aydemir Ö, Fişek G, Ölmez N, Memiş A. Kısa Form-36 (KF-36)'nın Türkçe Için Güvenilirliği ve Geçerliliği: Romatizmal Hastalığı Olan Bir Grup Hasta Ile Çalışma. Ilaç ve Tedavi Dergisi 1999;12:102-105. https://www.researchgate. net/publication/301799662_Kisa_Form-36_SF-36'nin_Turkce_ Versiyonunun_Guvenilirligi_ve_Gecerliligi_Reliability_and_Validity_ of_the_Turkish_Version_of_Short_Form-36_SF-36

9. Koçyiğit H, Aydemir Ö, Ölmez N, Fişek G, Ölmez N, Memiş A. Kısa Form-36 (SF-36)'nın Türkçe Versiyonunun Güvenilirliği ve Geçerliliği. Ilaç ve Tedavi Dergisi. 1999;12:102-106.

10. Aydemir Ö, Köroğlu E. Psikiyatride Kullanılan Klinik Ölçekler. Hekimler Yayın Birliği Ankara 2006;346-353.

11. Lam ET, Lam CL, Lai CL, Yuen MF, Fong DY, So TM. Healthrelated quality of life of Southern Chinese with chronic hepatitis B infection. Health Qual Life Outcomes. 2009;7:52.

12. Bondini S, Kallman J, Dan A, Younoszai Z, Ramsey L, Nader F, Younossi ZM. Health-related quality of life in patients with chronic hepatitis B. Liver Int. 2007;27:1119-1125.

13. Ozkan M, Corapçioglu A, Balcioglu I, Ertekin E, Khan S, Ozdemir S, Karayün D, Unsalver BO, Kocaman N, Kaymakglu S, Köroglu G. Psychiatric morbidity and its effect on the quality of life of patients with chronic hepatitis B and hepatitis C. Int J Psychiatry Med. 2006;36:283-297.

14. Fontana RJ, Moyer CA, Sonnad S, Lok ASF, Sneed-Pee N, Walsh $\mathrm{J}$, Klein S, Webster S. Comorbidities and quality of life in patients with interferon-refractory chronic hepatitis C. Am J Gastroenterol. 2001;96:170-178.

15. Teixeira MC, Ribeiro Mde F, Gayotto LC, Chamone Dde A, Strauss E. Worse quality of life in volunteer blood donors with hepatitis $C$. Transfusion. 2006;46:278-283.

16. Olson SH, Iyer S, Scott J, Erez O, Samuel S, Markovits T, Schwartz M, Toro C, Gambarin-Gelwan M, Kurtz RC. Cancer history and other personal factors affect quality of life in patients with hepatitis C. Health Qual Life Outcomes. 2005;3:39

17. Eşer I, Khorshid L, Türk G. Kronik Hepatit C Hastalarının Sağlıkla Illişkili Yaşam Kalitesinin Değerlendirilmesi. Viral Hepat J. 2006;11:41-45.

18. Kallman J, O'Neil MM, Larive B, Boparai N, Calabrese L, Younossi ZM. Fatigue and health-related quality of life (HROL) in chronic hepatitis C virus infection. Dig Dis Sci. 2007;52:2531-2539.

19. Braga SF, Peixoto SV, Gomes IC, Acúrcio Fde A, Andrade El, Cherchiglia ML. Factors associated with health-related quality of life in elderly patients on hemodialysis. Rev Saude Publica. 2011:45:1127-1136

20. Gao F, Gao R, Li G, Shang ZM, Hao JY. Health-related quality of life and survival in Chinese patients with chronic liver disease. Health Qual Life Outcomes. 2013;11:131.

21. Vlassoff C. Gender differences in determinants and consequences of health and illness. J Health Popul Nutr. 2007;25:47-61.

22. Hussain KB, Fontana RJ, Moyer CA, Su GL, Sneed-Pee N, Lok AS. Comorbid illness is an important determinant of healthrelated quality of life in patients with chronic hepatitis C. Am J Gastroenterol. 2001;96:2737-2744.

23. Gjeruldsen S, Loge JH, Myrvang B, Opjordsmoen S. Drug addiction in hepatitis $C$ patients leads to a lower quality of life. Nord J Psychiatry. 2006;60:157-161.

24. Spiegel BM, Younossi ZM, Hays RD, Revicki D, Robbins $S$, Kanwal F. Impact of hepatitis $C$ on health related quality of life: a systematic review and quantitative assessment. Hepatology. 2005; $41: 790-800$

25. Kang SC, Hwang SJ, Lee SH, Chang FY, Lee SD. Health-related quality of life and impact of antiviral treatment in Chinese patients with chronic hepatitis C in Taiwan. World J Gastroenterol. 2005; 11:7494-7498.

26. Gallegos-Orozco JF, Fuentes AP, Gerardo Argueta J, Pérez-Pruna C, Hinojosa-Becerril C, Sixtos-Alonso MS, Cruz-Castellanos S, Gutiérrez-Reyes G, Olivera-Martínez MA, Gutiérrez-Ruiz MC, Kershenobich D. Health-related quality of life and depression in patients with chronic hepatitis C. Arch Med Res. 2003;34:124129.

27. Pojoga C, Dumitraşcu DL, Pascu O, Grigorescu M, Radu C, Damian D. Impaired health-related quality of life in Romanian patients with chronic viral hepatitis before antiviral therapy. Eur $\mathrm{J}$ Gastroenterol Hepatol. 2004;16:27-31.

28. Bernstein D, Kleinman L, Barker CM, Revicki DA, Green J. Relationship of health-related quality of life to treatment adherence and sustained response in chronic hepatitis $\mathrm{C}$ patients. Hepatology. 2002;35:704-708.

29. McHutchison JG, Ware JE Jr, Bayliss MS, Pianko S, Albrecht JK, Cort S, Yang I, Neary MP; Hepatitis Interventional Therapy Group. The effects of interferon alpha-2b in combination with ribavirin on health related quality of life and work productivity. J Hepatol. 2001;34:140-147.

30. Marcellin P, Lau GK, Zeuzem S, Heathcote EJ, Pockros PJ, Reddy KR, Piratvisuth T, Farci P, Chow WC, Jia JD, Paik W, Wintfeld N, Pluck N. Comparing the safety, tolerability and quality of life in patients with chronic hepatitis B vs chronic hepatitis C treated with peginterferon alpha-2a. Liver Int. 2008;28:477-485. 